Supporting Information

\title{
Synthesis, Structure, and Hydrogenolysis of Pyridine Dicarbene Pincer Iron Dialkyl Complexes.
}

Stephan M. Rummelt, Jonathan M. Darmon, Renyuan Pony Yu, Peter Viereck, Tyler Pabst, Zoë R. Turner, Grant W. Margulieux, Shunlin Gu, Paul J. Chirik*

Department of Chemistry, Frick Laboratory

Princeton University, Princeton, NJ 08544, USA

pchirik@princeton.edu

\section{Table of Contents}

Additional Spectroscopic Data 


\section{Additional Spectroscopic Data}

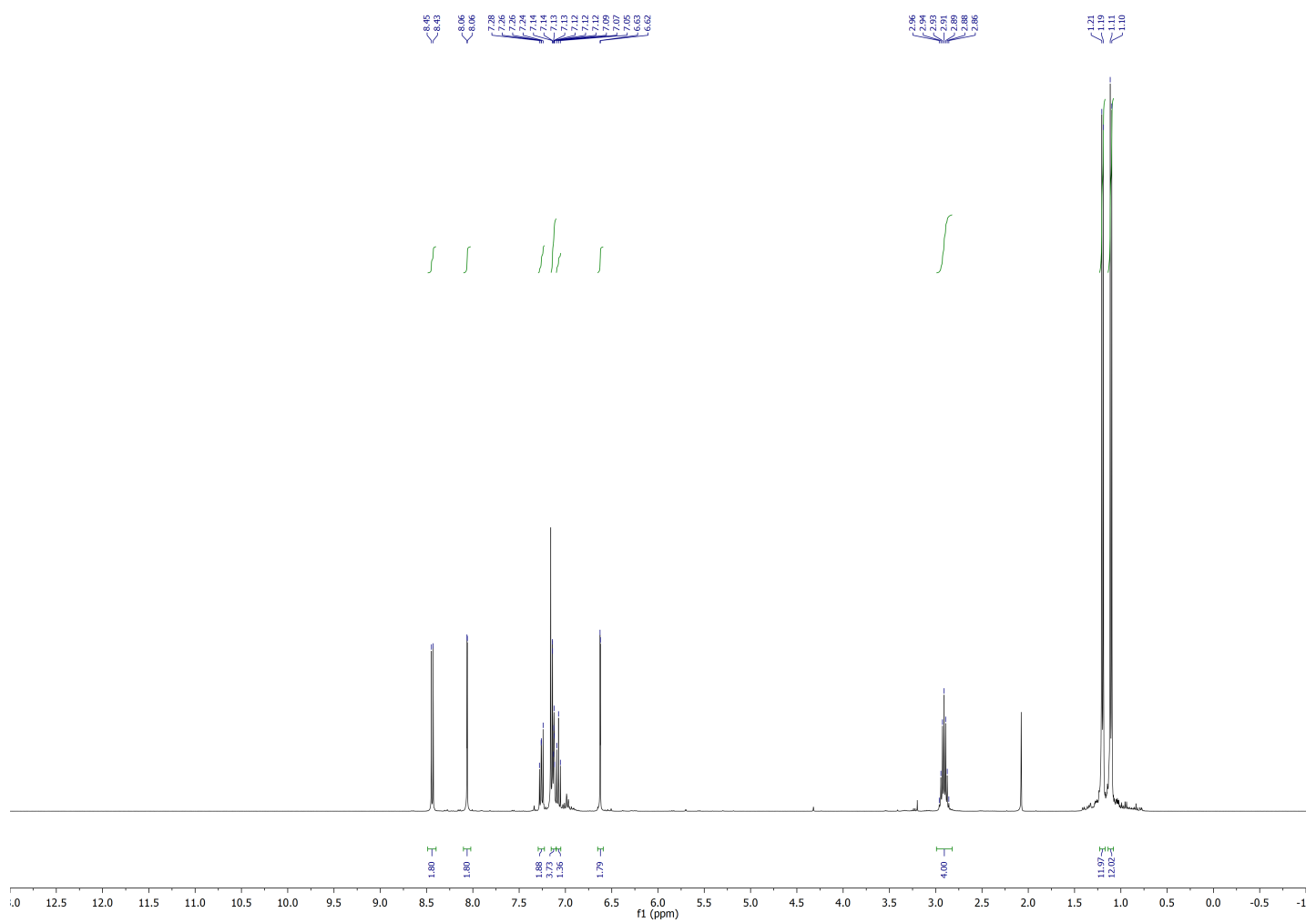

Figure S1. ${ }^{1} \mathrm{H}$ NMR spectrum of ${ }^{\mathrm{iPr}} \mathrm{CNC}$ in $\mathrm{C}_{6} \mathrm{D}_{6}$ at $25^{\circ} \mathrm{C}$.

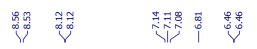

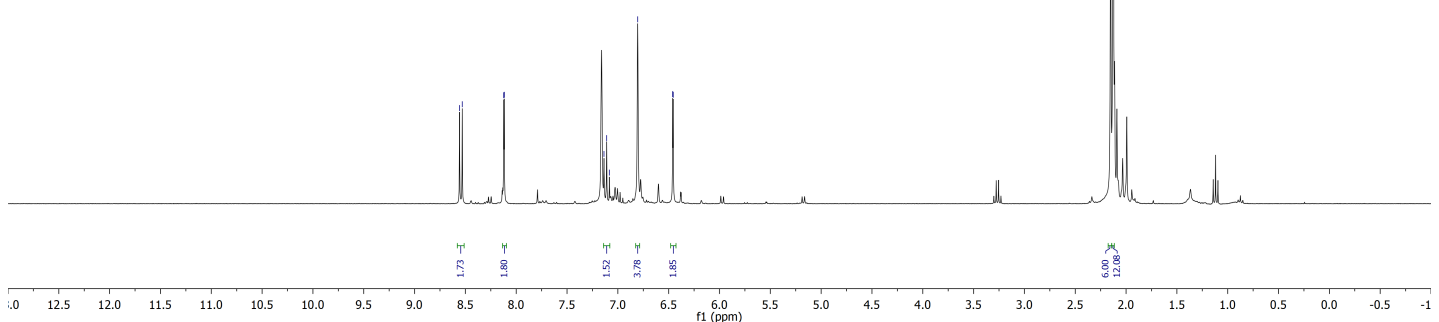

Figure S2. ${ }^{15} \mathrm{C}$ NMR spectrum of ${ }^{1 \mathrm{Ir}} \mathrm{CNC}$ in $\mathrm{C}_{6} \mathrm{D}_{6}$ at $25^{\circ} \mathrm{C}$. 


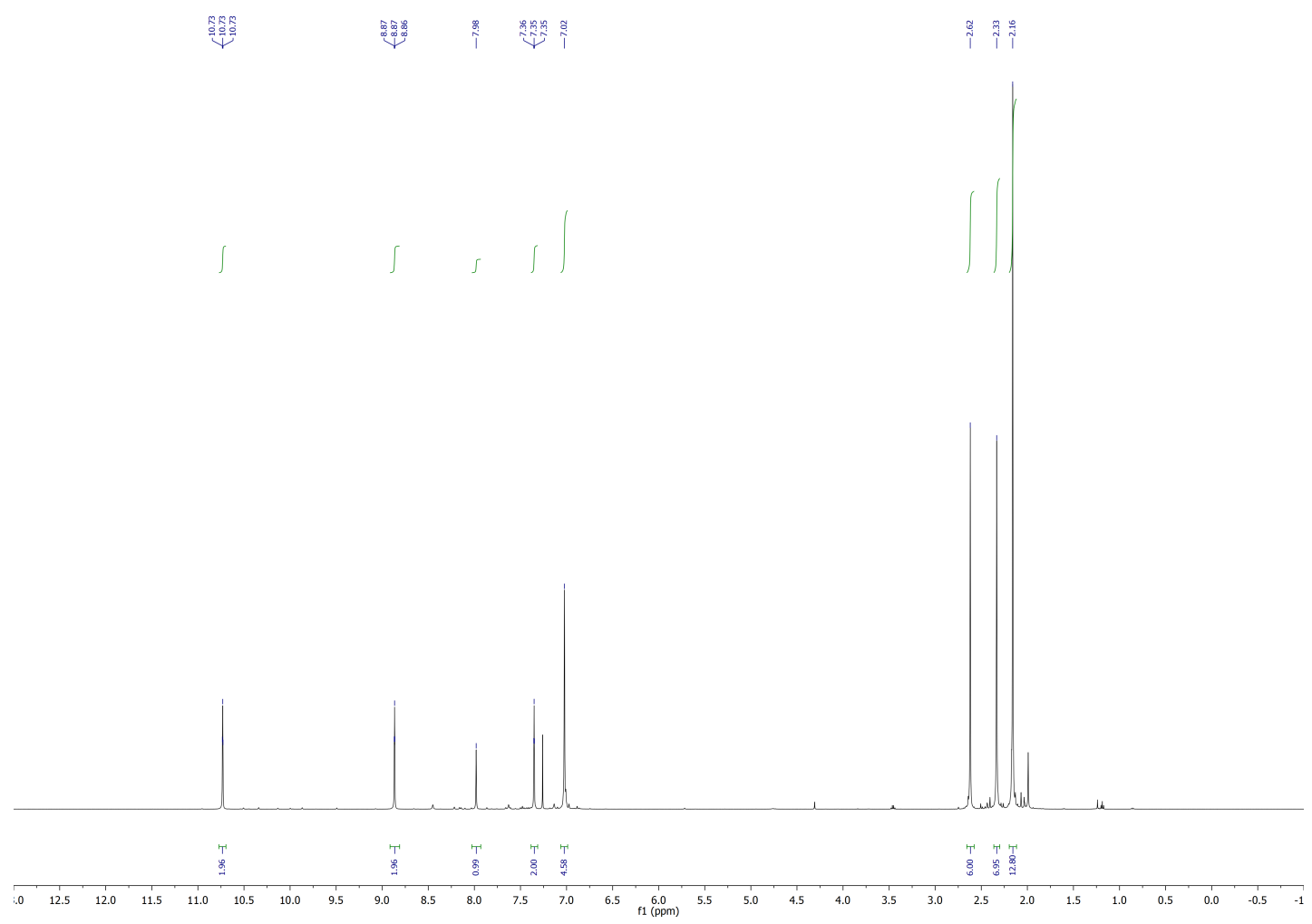

Figure S3. ${ }^{1} \mathrm{H}$ NMR spectrum of $3,5-\mathrm{Me}_{2}{ }^{\mathrm{Mes}} \mathrm{CNC}(\mathrm{HI})_{2}$ in $\mathrm{CDCl}_{3}$ at $25^{\circ} \mathrm{C}$.
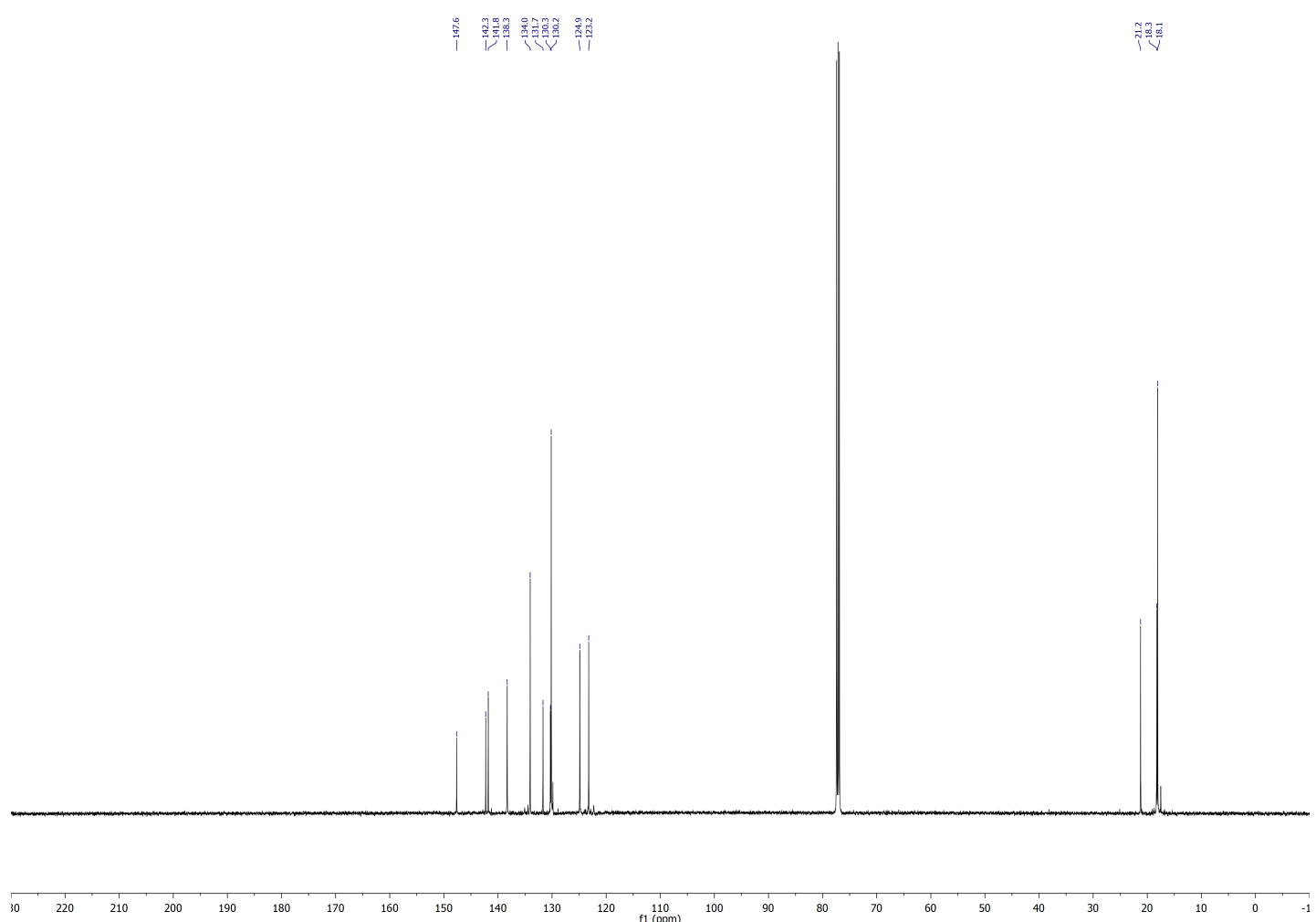

Figure S4. ${ }^{13} \mathrm{C}$ NMR spectrum of $3,5-\mathrm{Me}_{2}{ }^{\mathrm{Mes}} \mathrm{CNC}(\mathrm{HI})_{2}$ in $\mathrm{CDCl}_{3}$ at $25^{\circ} \mathrm{C}$. 


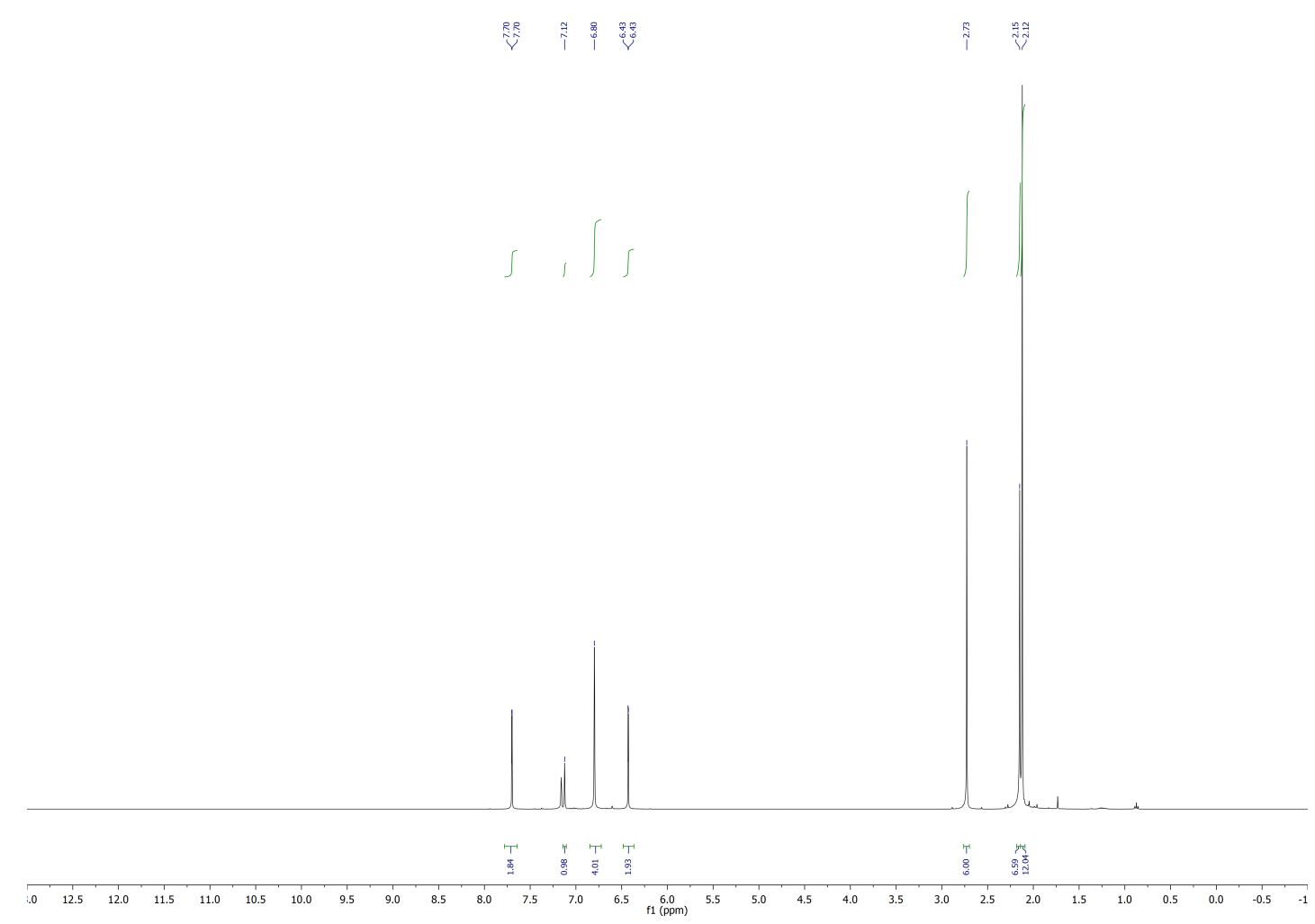

Figure S5. ${ }^{1} \mathrm{H}$ NMR spectrum of $3,5-\mathrm{Me}_{2}{ }^{\mathrm{Mes}} \mathrm{CNC}$ in $\mathrm{C}_{6} \mathrm{D}_{6}$ at $25^{\circ} \mathrm{C}$.
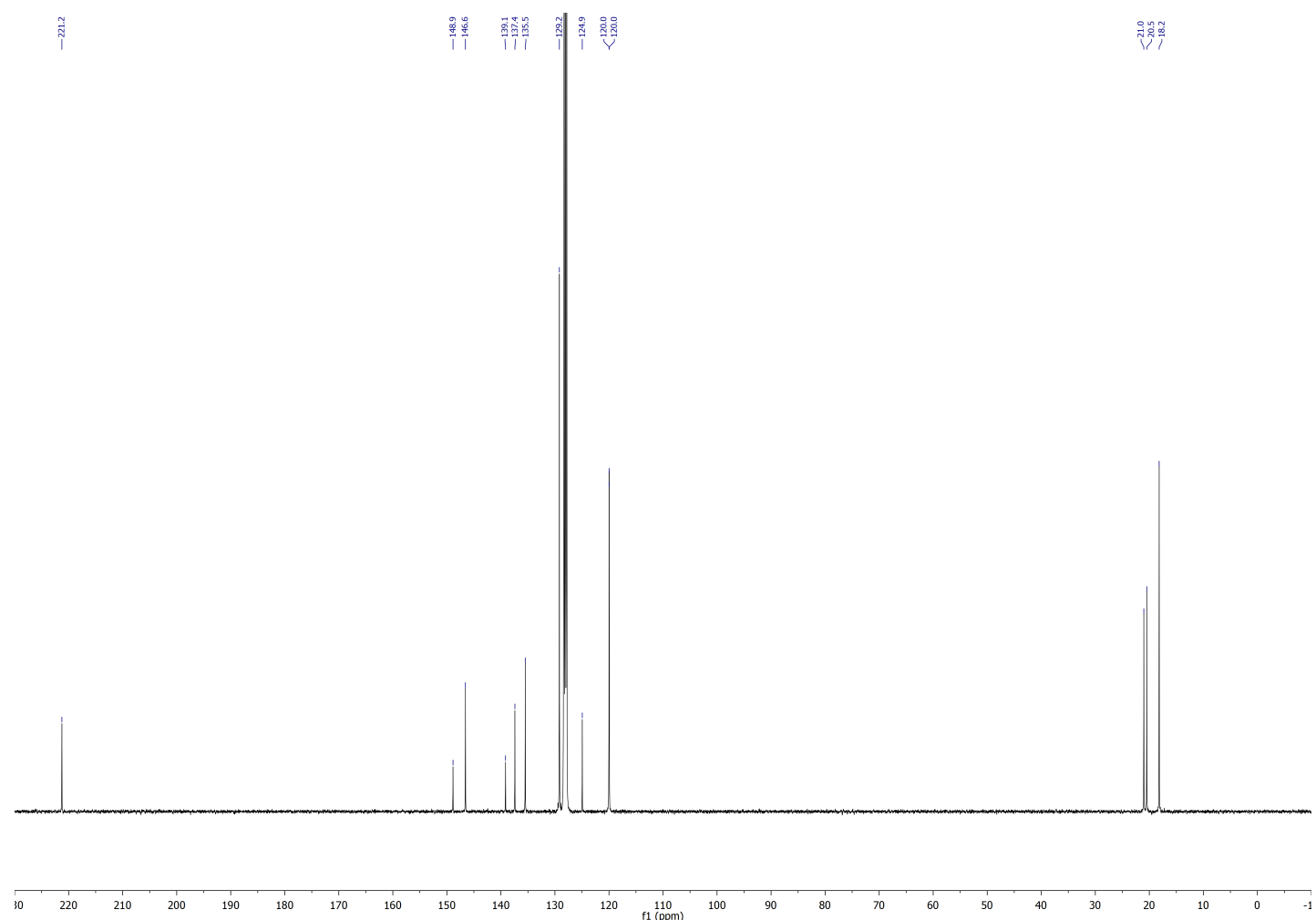

Figure S6. ${ }^{13} \mathrm{C}$ NMR spectrum of $3,5-\mathrm{Me}_{2}{ }^{\mathrm{Mes}} \mathrm{CNC}$ in $\mathrm{C}_{6} \mathrm{D}_{6}$ at $25^{\circ} \mathrm{C}$. 


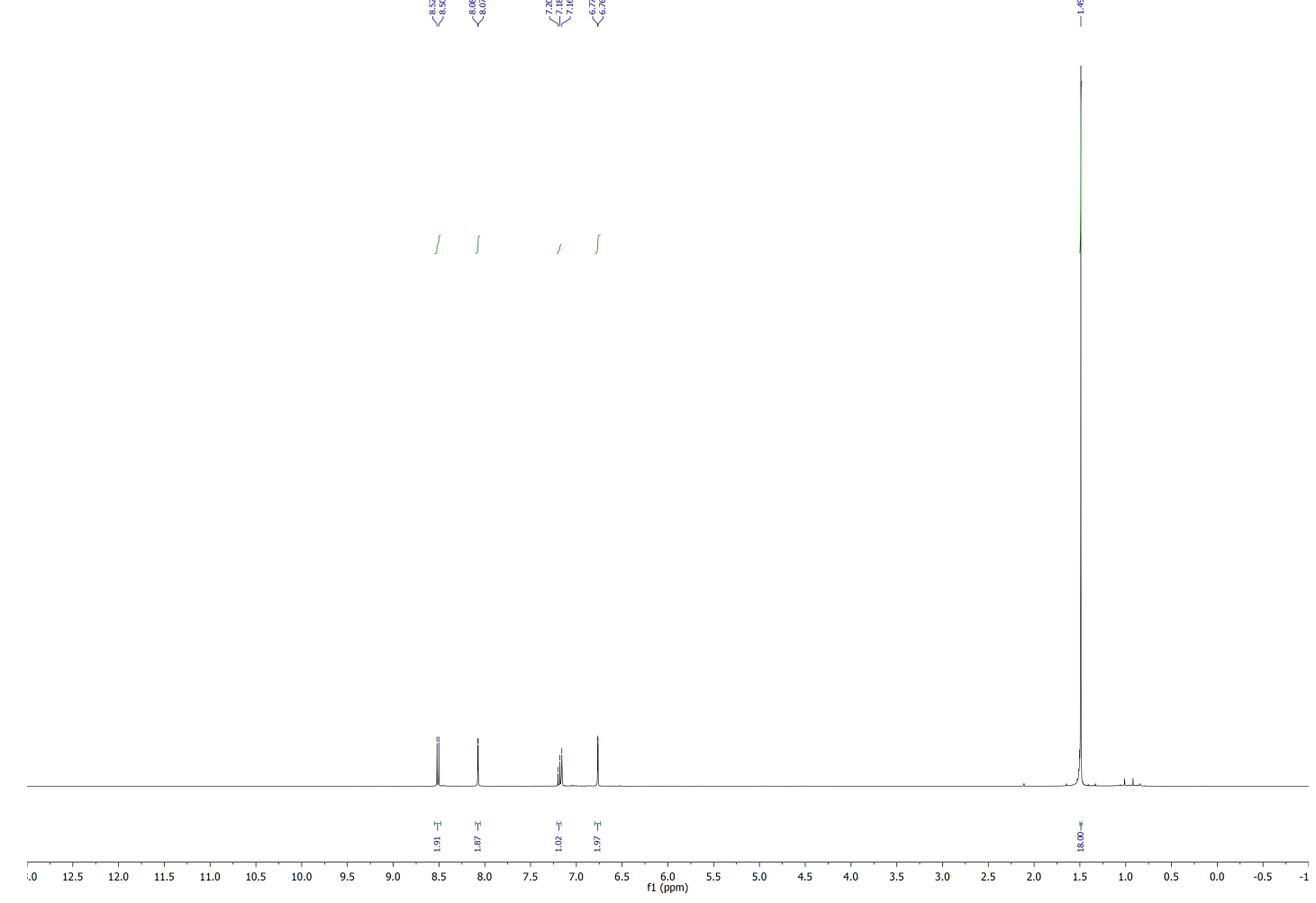

Figure S7. ${ }^{1} \mathrm{H}$ NMR spectrum of ${ }^{\mathrm{tBu}} \mathrm{ACNC}$ in $\mathrm{C}_{6} \mathrm{D}_{6}$ at $25^{\circ} \mathrm{C}$.
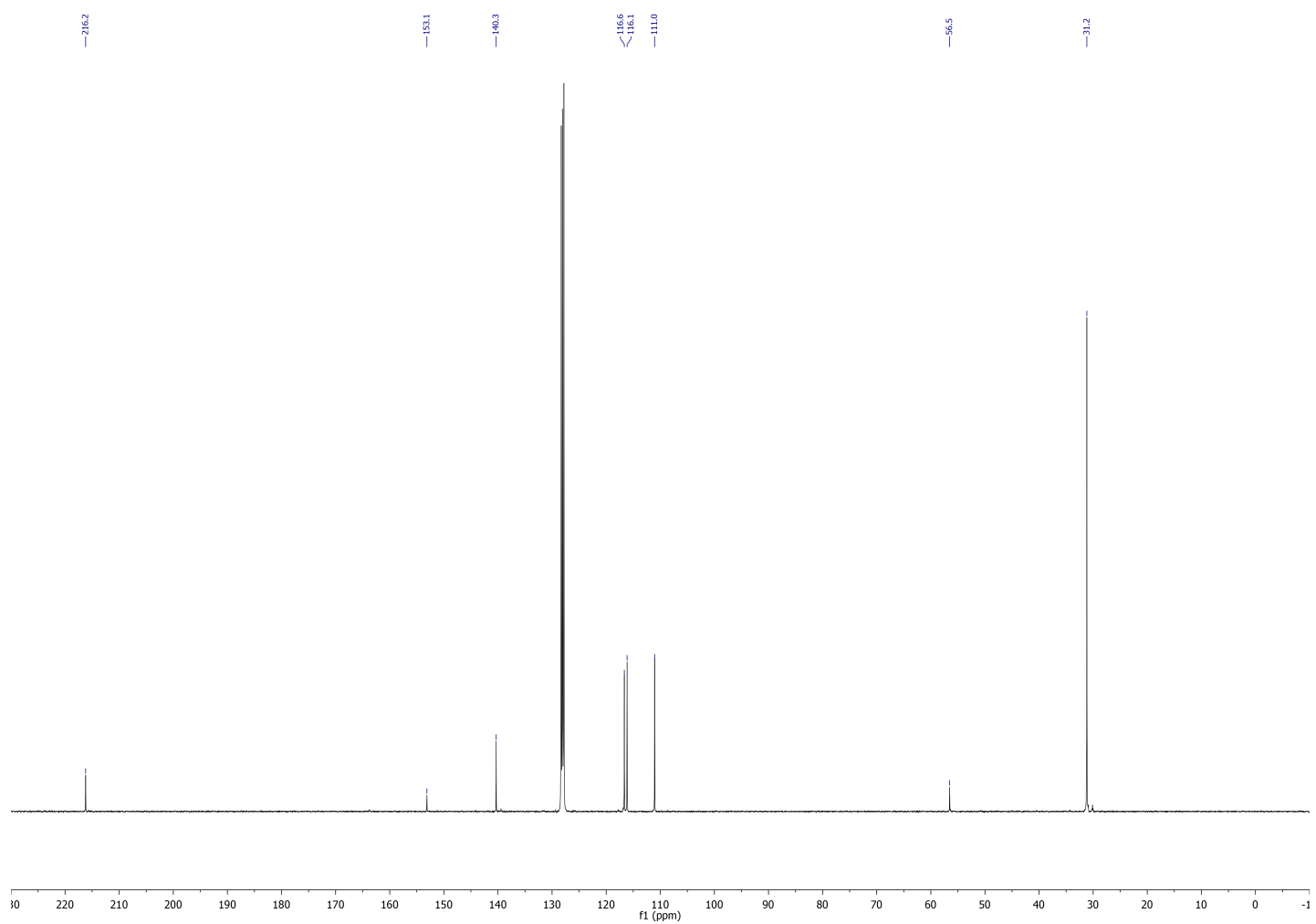

Figure S8. ${ }^{13} \mathrm{C}$ NMR spectrum of ${ }^{\mathrm{tBu}} \mathrm{ACNC}$ in $\mathrm{C}_{6} \mathrm{D}_{6}$ at $25^{\circ} \mathrm{C}$. 


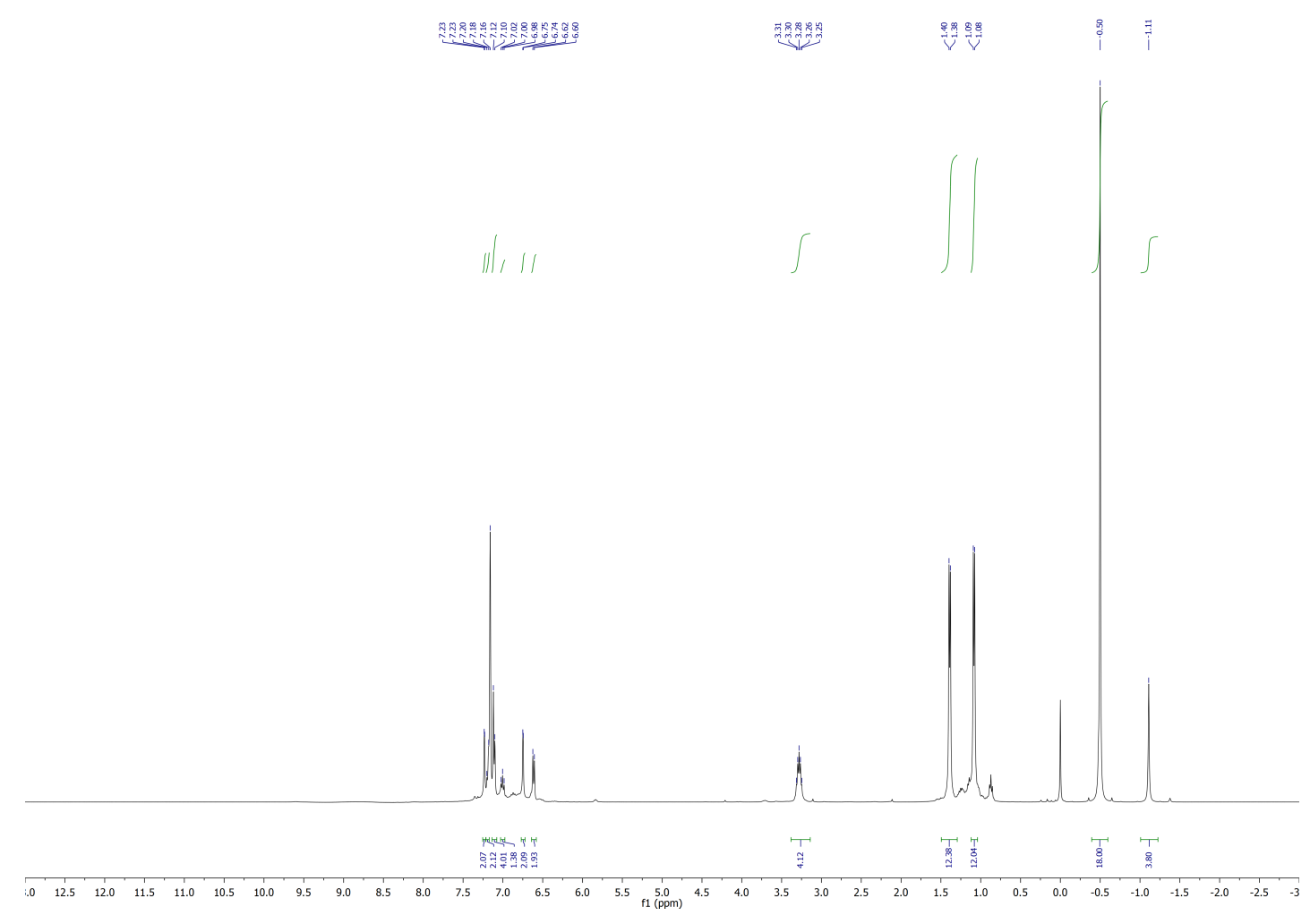

Figure S9. ${ }^{1} \mathrm{H}$ NMR spectrum of $\left({ }^{\mathrm{iPr}} \mathrm{CNC}\right) \mathrm{Fe}\left(\mathrm{CH}_{2} \mathrm{SiMe}_{3}\right)_{2}\left(\mathrm{~N}_{2}\right)$ in $\mathrm{C}_{6} \mathrm{D}_{6}$ at $25^{\circ} \mathrm{C}$.
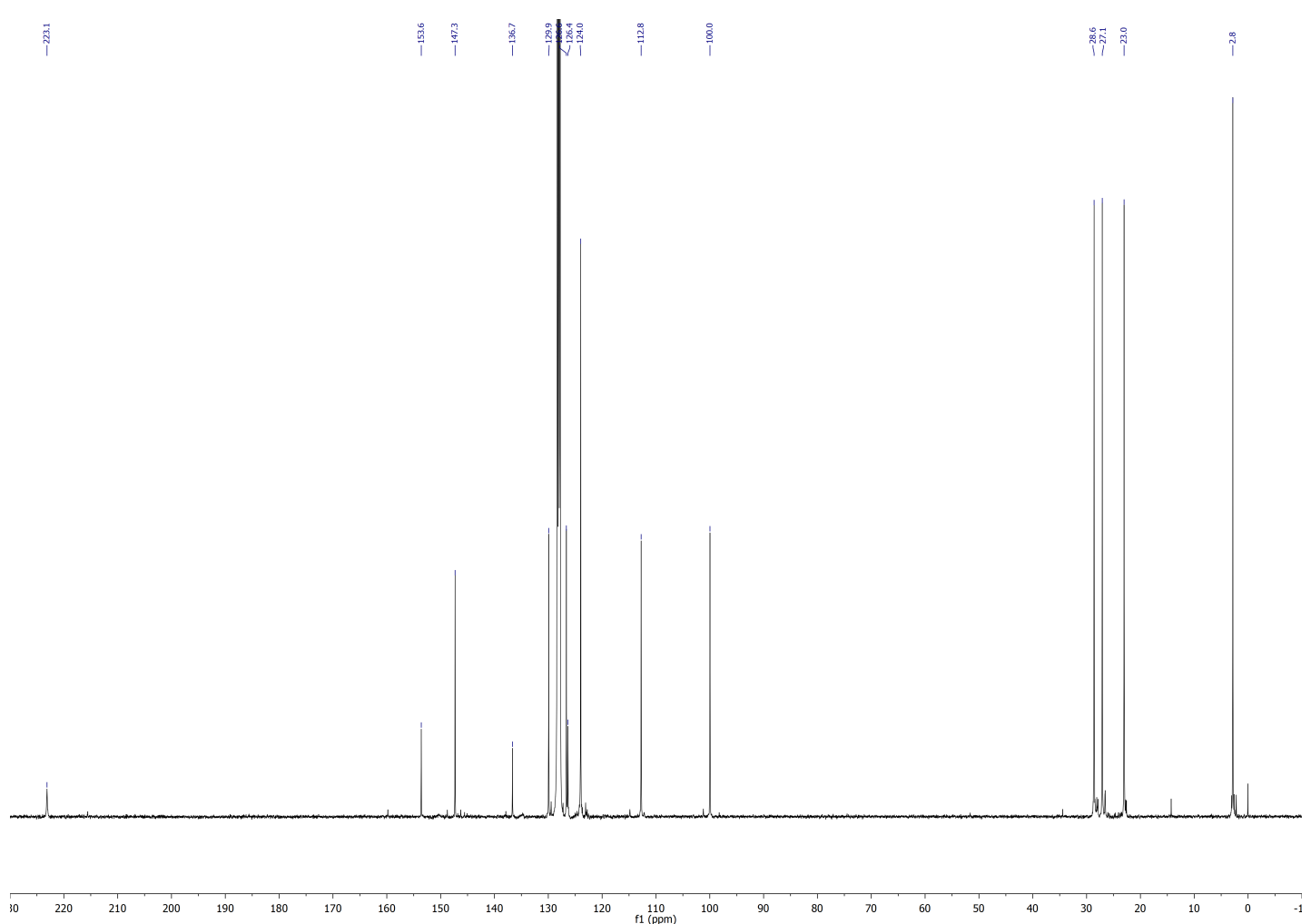

Figure S10. ${ }^{13} \mathrm{C}$ NMR spectrum of $\left({ }^{\mathrm{iPr}} \mathrm{CNC}\right) \mathrm{Fe}\left(\mathrm{CH}_{2} \mathrm{SiMe}_{3}\right)_{2}\left(\mathrm{~N}_{2}\right)$ in $\mathrm{C}_{6} \mathrm{D}_{6}$ at $25^{\circ} \mathrm{C}$. 


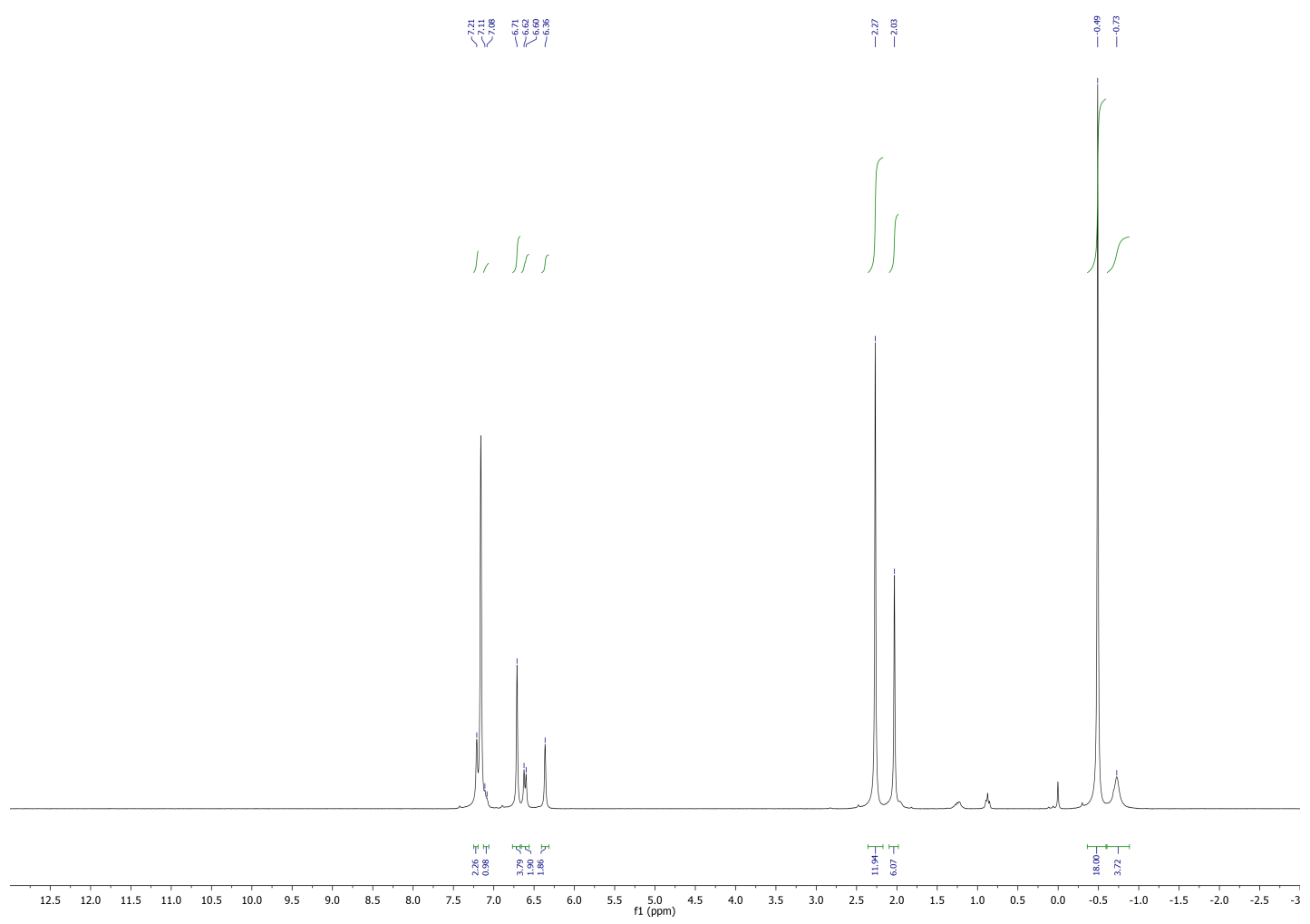

Figure S11. ${ }^{1} \mathrm{H}$ NMR spectrum of $\left({ }^{\mathrm{Mes}} \mathrm{CNC}\right) \mathrm{Fe}\left(\mathrm{CH}_{2} \mathrm{SiMe}_{3}\right)_{2}\left(\mathrm{~N}_{2}\right)$ in $\mathrm{C}_{6} \mathrm{D}_{6}$ at $25^{\circ} \mathrm{C}$.
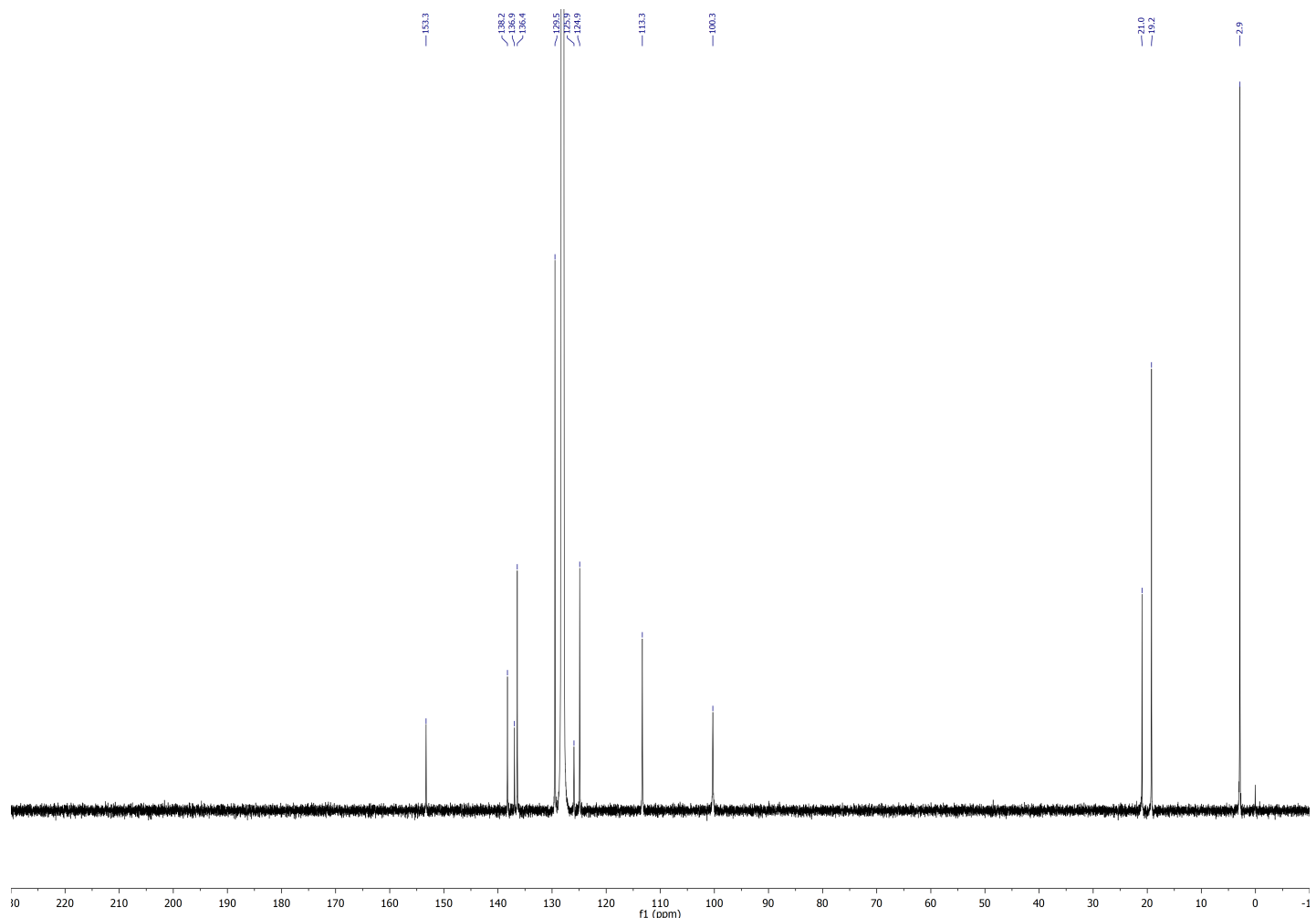

Figure S12. ${ }^{13} \mathrm{C}$ NMR spectrum of $\left({ }^{\mathrm{Mes}} \mathrm{CNC}\right) \mathrm{Fe}\left(\mathrm{CH}_{2} \mathrm{SiMe}_{3}\right)_{2}\left(\mathrm{~N}_{2}\right)$ in $\mathrm{C}_{6} \mathrm{D}_{6}$ at $25{ }^{\circ} \mathrm{C}$. 


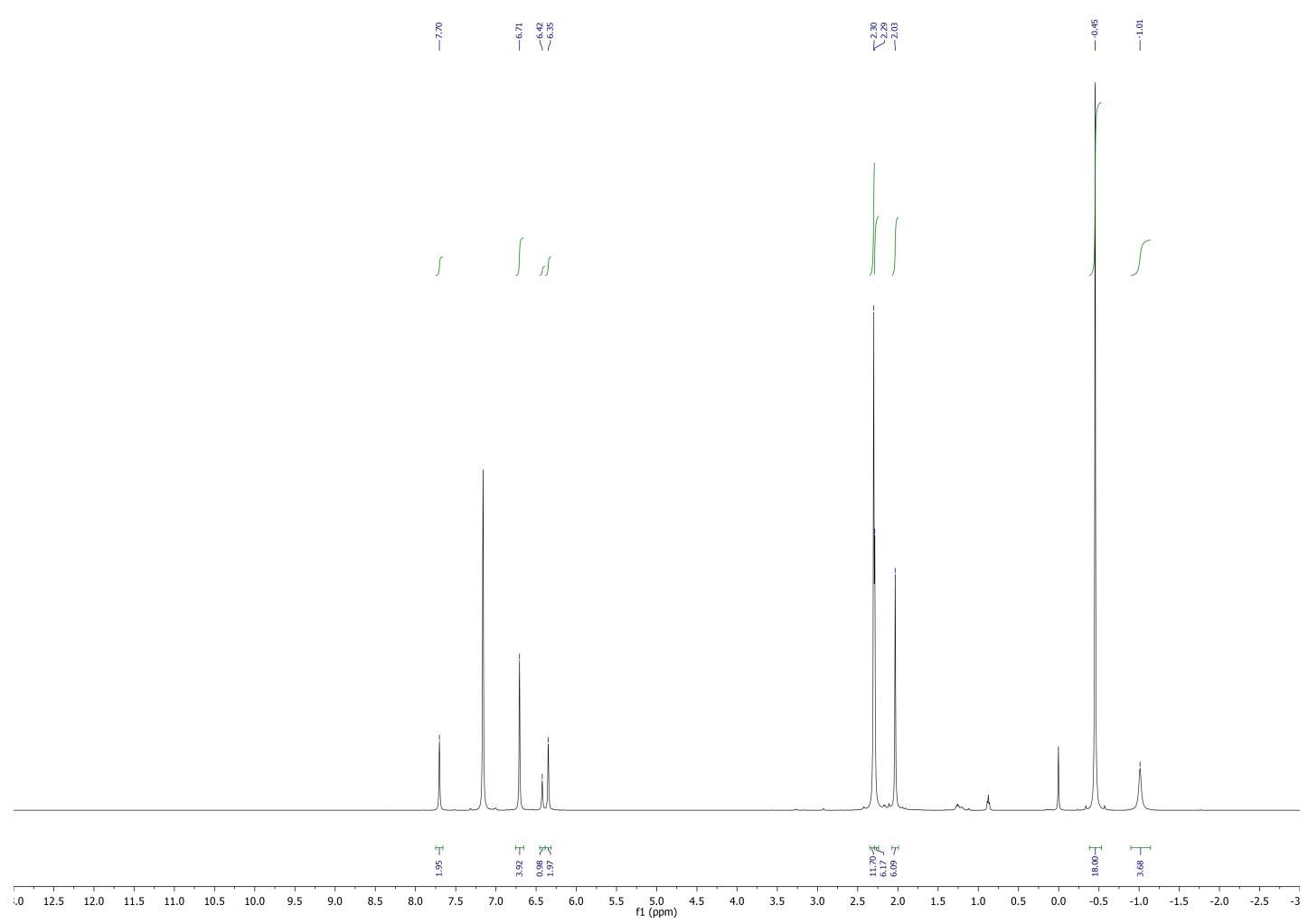

Figure S13. ${ }^{1} \mathrm{H}$ NMR spectrum of $\left(3,5-\mathrm{Me}_{2}{ }^{\mathrm{Mes}} \mathrm{CNC}\right) \mathrm{Fe}\left(\mathrm{CH}_{2} \mathrm{SiMe}_{3}\right)_{2}\left(\mathrm{~N}_{2}\right)$ in $\mathrm{C}_{6} \mathrm{D}_{6}$ at $25{ }^{\circ} \mathrm{C}$.
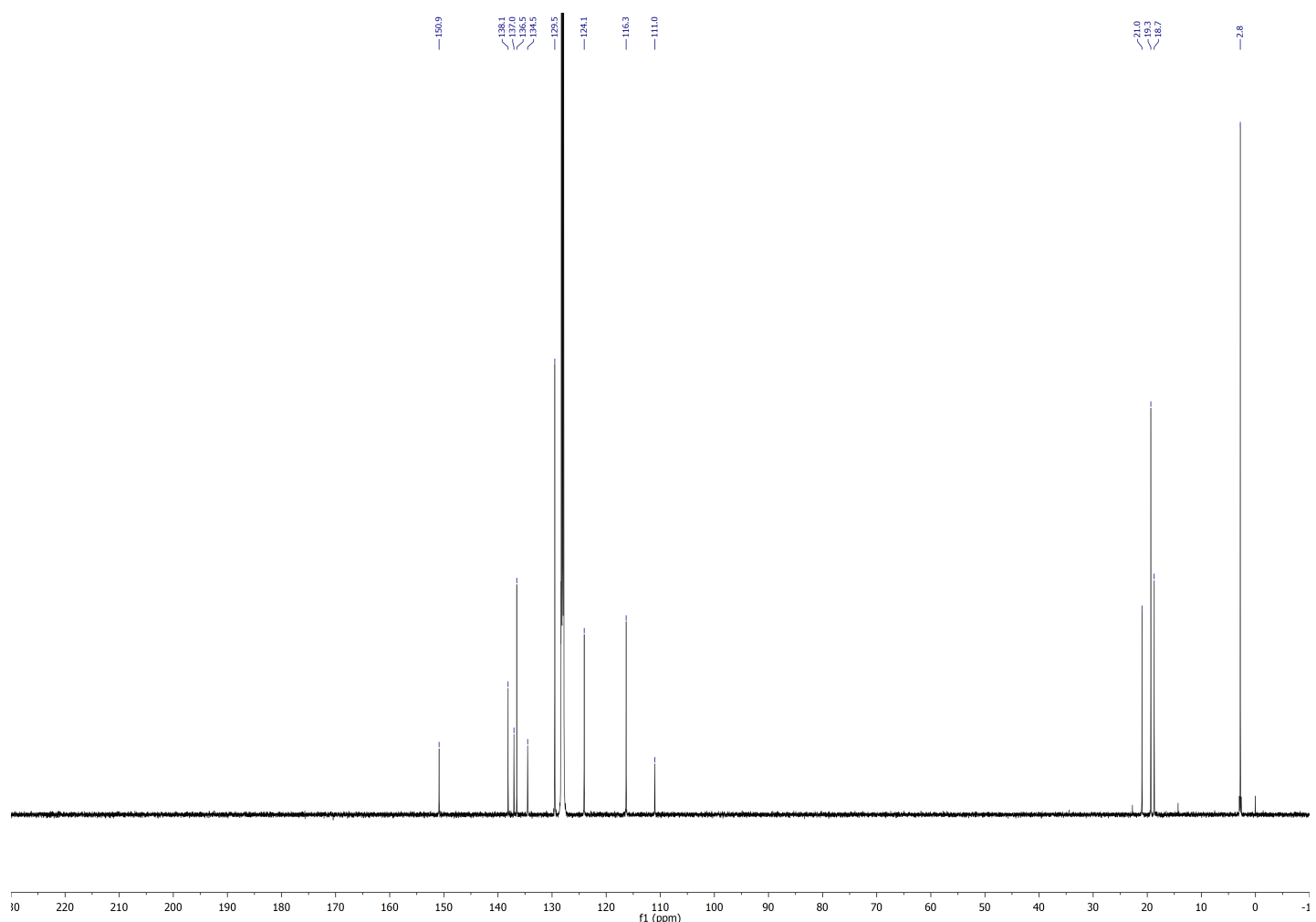

Figure S14. ${ }^{13} \mathrm{C}$ NMR spectrum of $\left(3,5-\mathrm{Me}_{2}{ }^{\mathrm{Mes}} \mathrm{CNC}\right) \mathrm{Fe}\left(\mathrm{CH}_{2} \mathrm{SiMe}_{3}\right)_{2}\left(\mathrm{~N}_{2}\right)$ in $\mathrm{C}_{6} \mathrm{D}_{6}$ at $25^{\circ} \mathrm{C}$. 


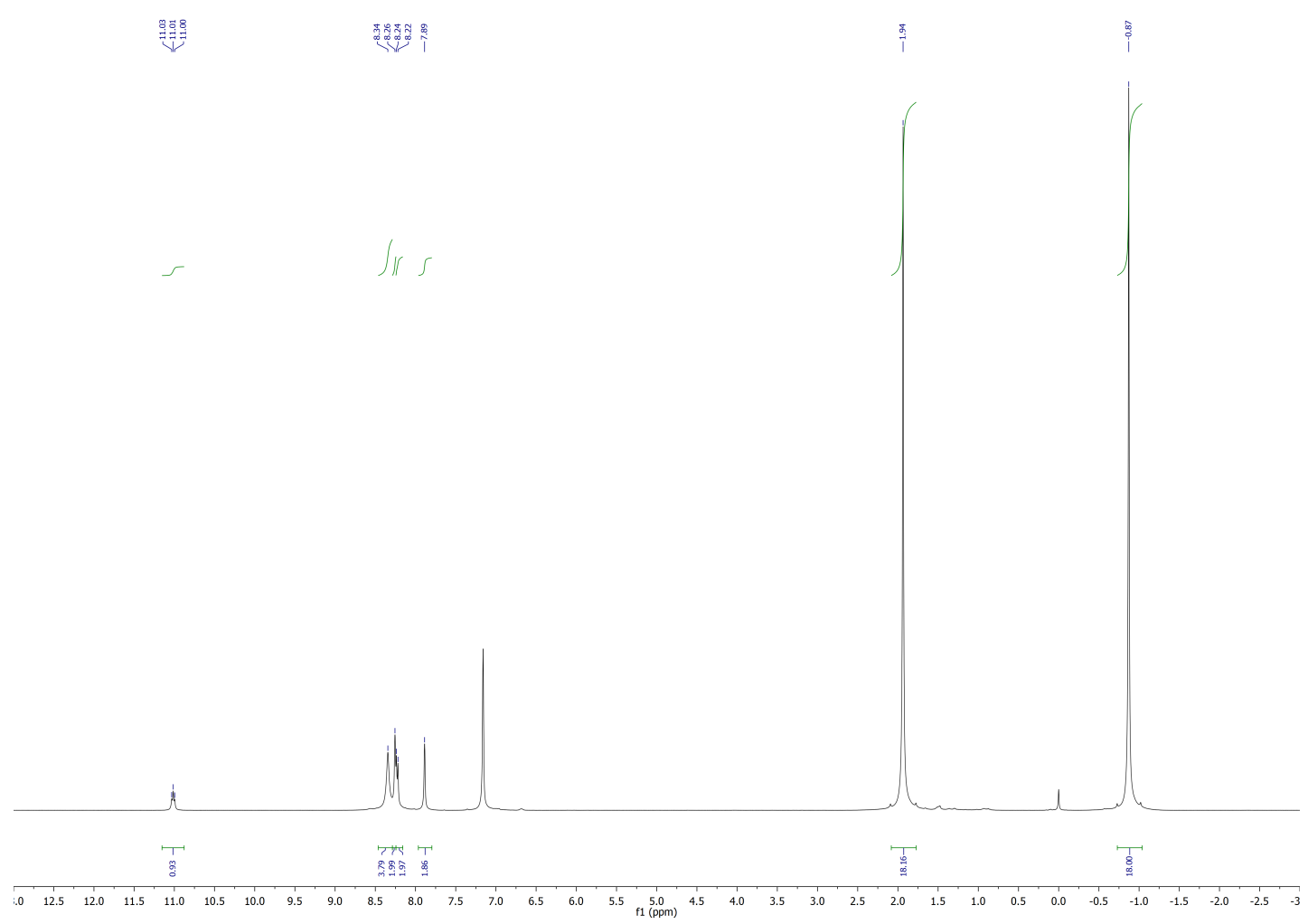

Figure S15. ${ }^{1} \mathrm{H}$ NMR spectrum of $\left({ }^{\mathrm{tBu}} \mathrm{ACNC}\right) \mathrm{Fe}\left(\mathrm{CH}_{2} \mathrm{SiMe}_{3}\right)_{2}\left(\mathrm{~N}_{2}\right)$ in $\mathrm{C}_{6} \mathrm{D}_{6}$ at $25^{\circ} \mathrm{C}$.
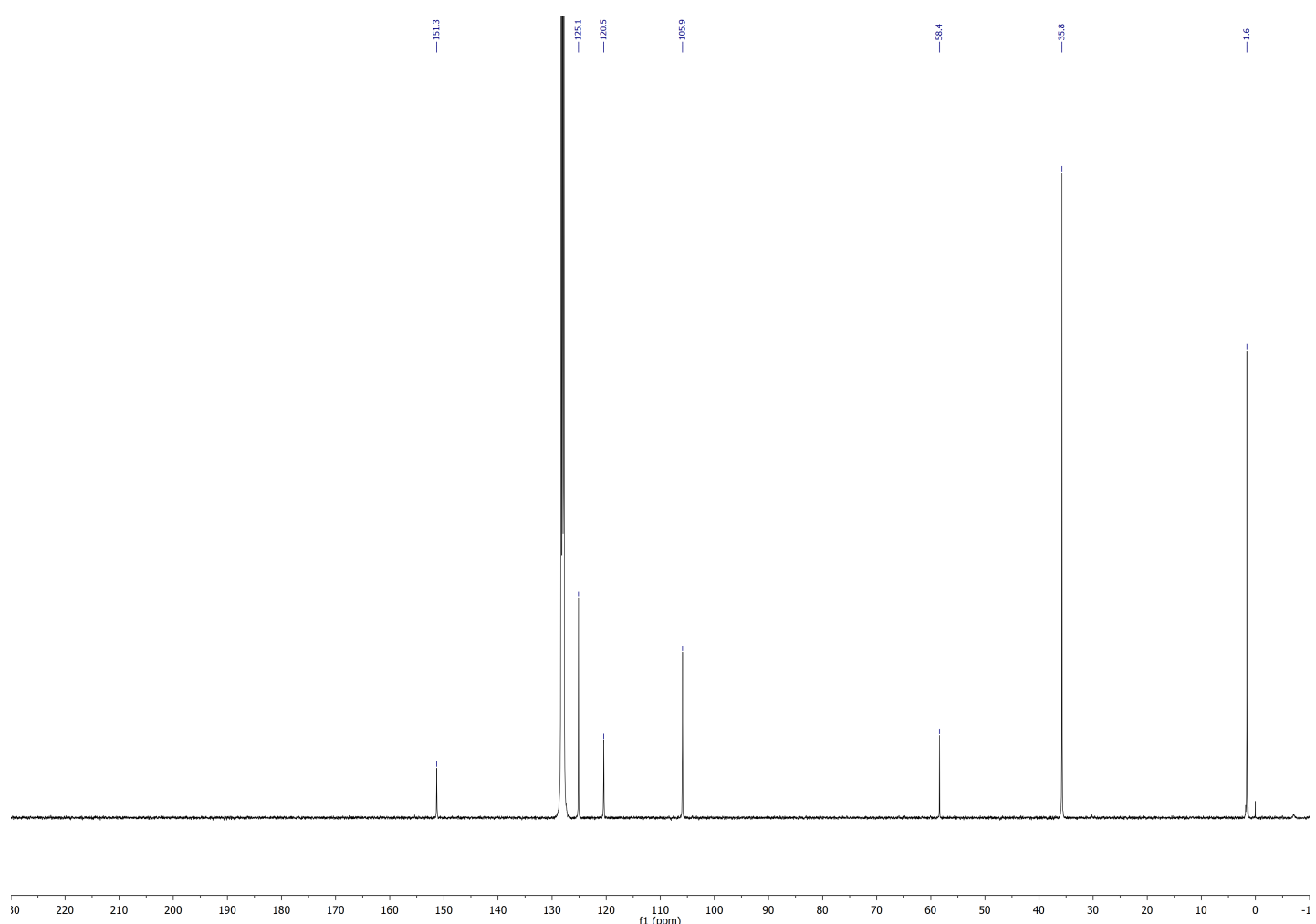

Figure S16. ${ }^{13} \mathrm{C}$ NMR spectrum of $\left({ }^{\mathrm{tBu}} \mathrm{ACNC}\right) \mathrm{Fe}\left(\mathrm{CH}_{2} \mathrm{SiMe}_{3}\right)_{2}\left(\mathrm{~N}_{2}\right)$ in $\mathrm{C}_{6} \mathrm{D}_{6}$ at $25{ }^{\circ} \mathrm{C}$. 


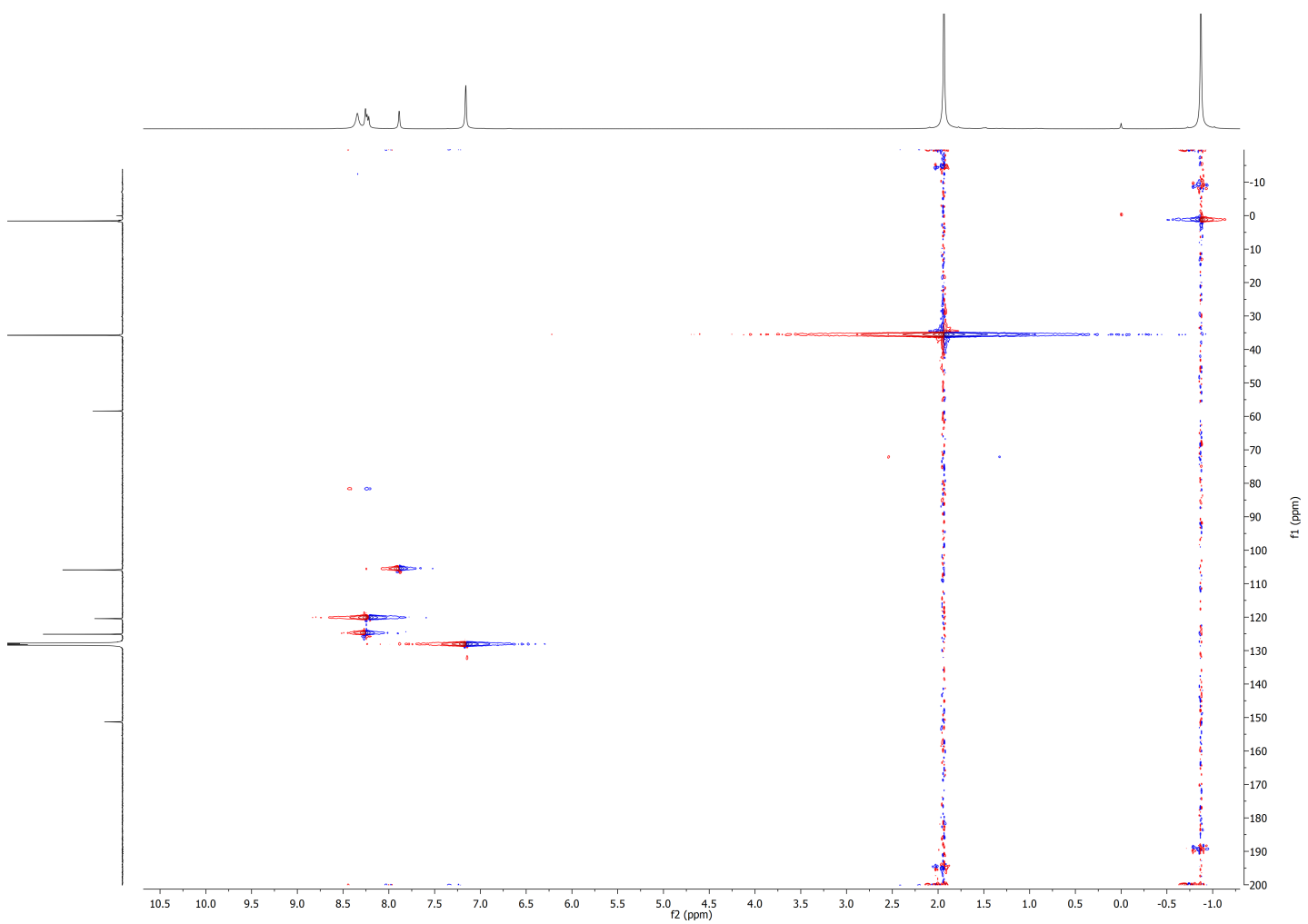

Figure S17. HSQC NMR spectrum of $\left({ }^{\mathrm{tBu}} \mathrm{ACNC}\right) \mathrm{Fe}\left(\mathrm{CH}_{2} \mathrm{SiMe}_{3}\right)_{2}\left(\mathrm{~N}_{2}\right)$ in $\mathrm{C}_{6} \mathrm{D}_{6}$ at $25^{\circ} \mathrm{C}$.

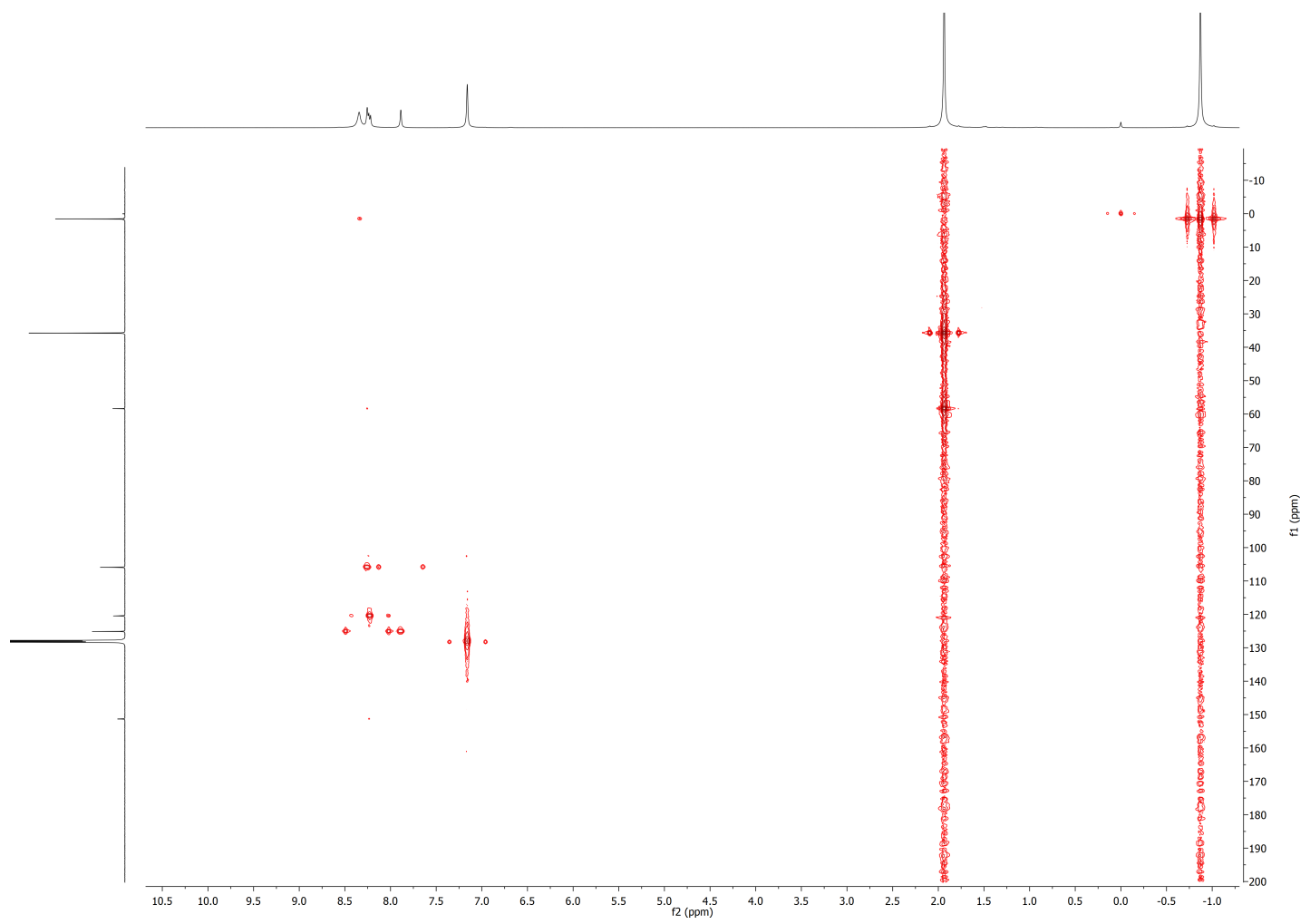

Figure S18. HMBC NMR spectrum of $\left({ }^{\mathrm{tBu}} \mathrm{ACNC}\right) \mathrm{Fe}\left(\mathrm{CH}_{2} \mathrm{SiMe}_{3}\right)_{2}\left(\mathrm{~N}_{2}\right)$ in $\mathrm{C}_{6} \mathrm{D}_{6}$ at $25^{\circ} \mathrm{C}$. 


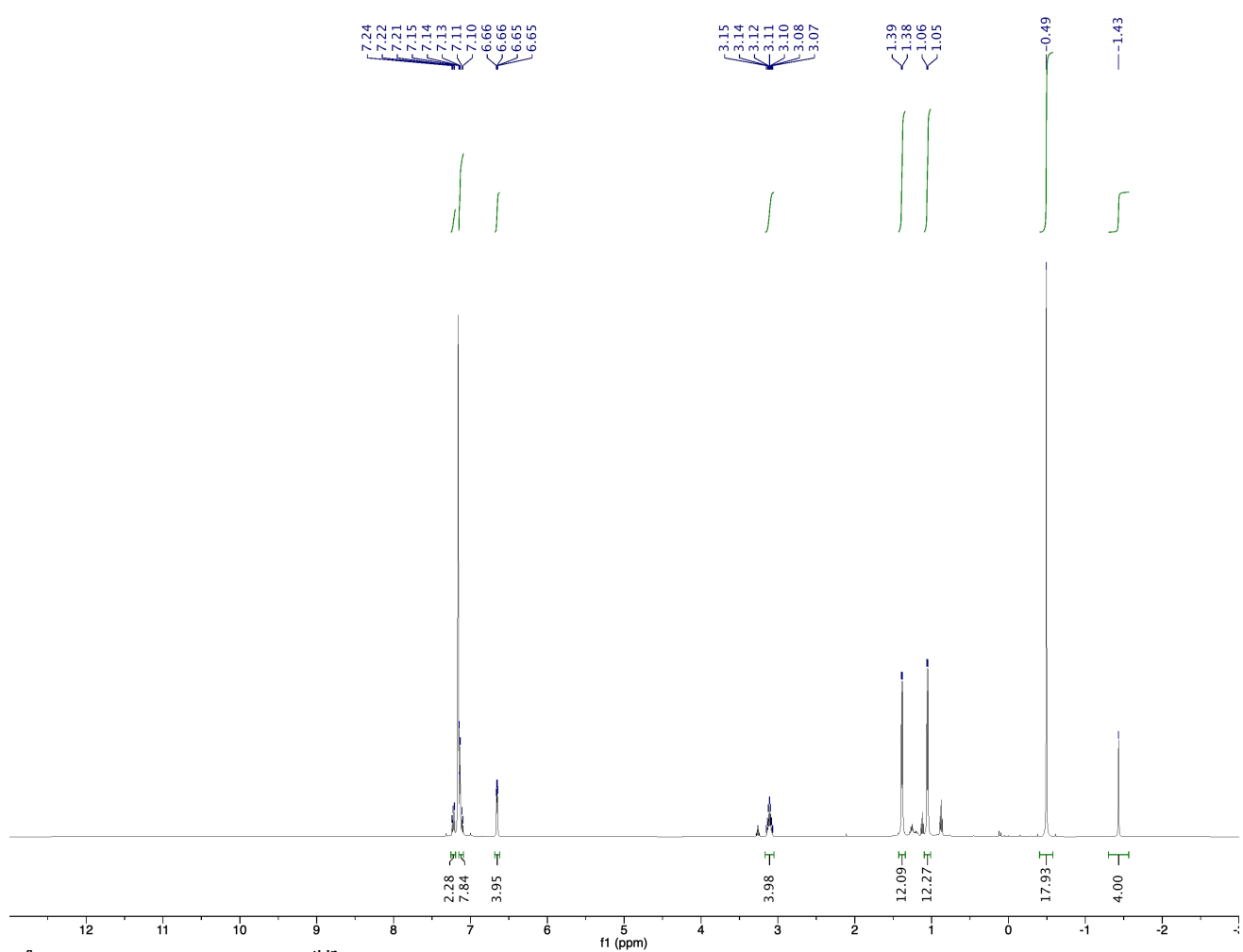

Figure S19. ${ }^{~} \mathrm{H}$ NMR spectrum of $\left({ }^{1+\mathrm{r}} \mathrm{CNC}\right) \mathrm{Fe}\left(\mathrm{CH}_{2} \mathrm{SiMe}_{3}\right)_{2}(\mathrm{CO})$ in $\mathrm{C}_{6} \mathrm{D}_{6}$ at $25^{\circ} \mathrm{C}$.
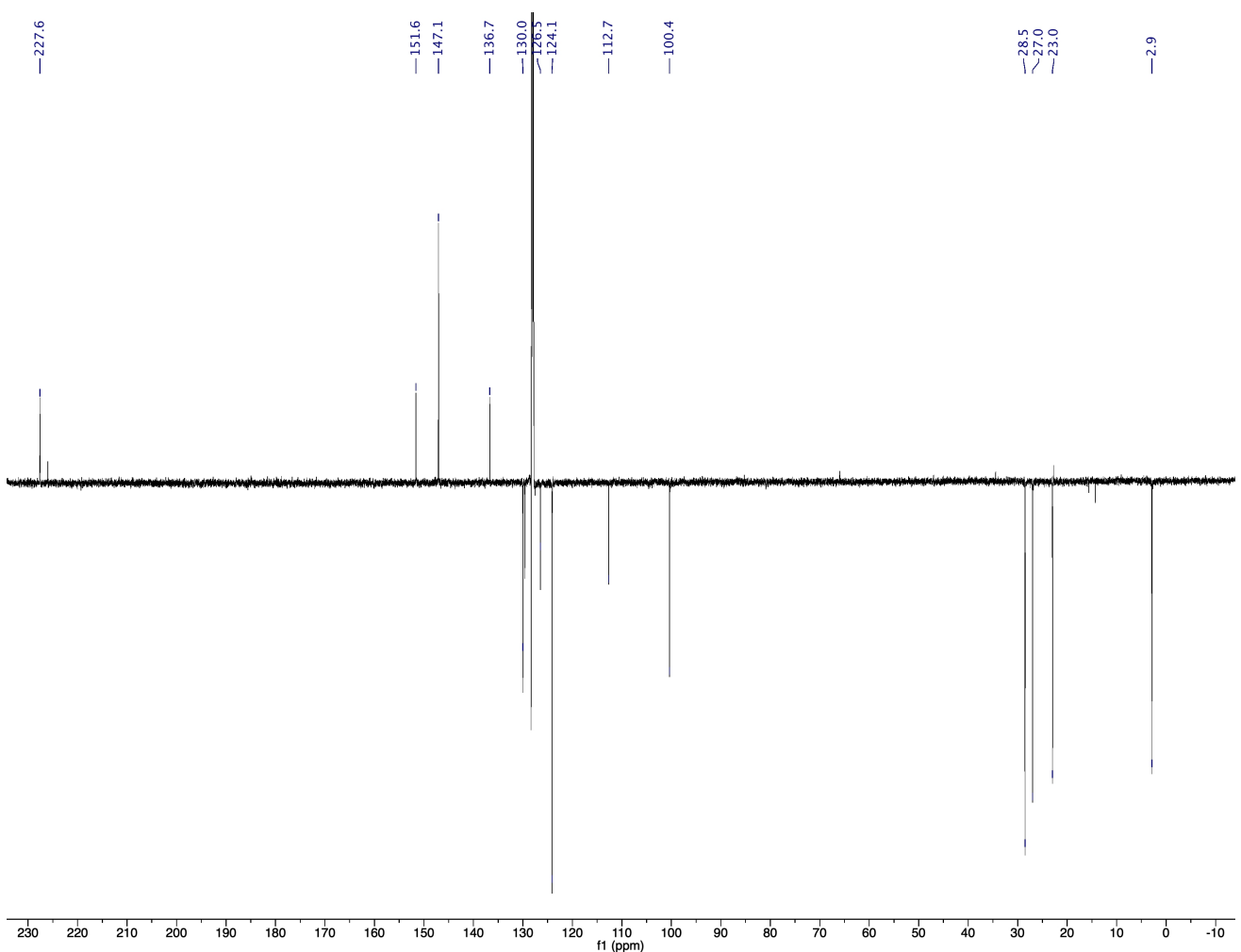

Figure S20. ${ }^{13} \mathrm{C}$ APT NMR spectrum of $\left({ }^{(\mathrm{Hr}} \mathrm{CNC}\right) \mathrm{Fe}\left(\mathrm{CH}_{2} \mathrm{SiMe}_{3}\right)_{2}(\mathrm{CO})$ in $\mathrm{C}_{6} \mathrm{D}_{6}$ at $25{ }^{\circ} \mathrm{C}$. 


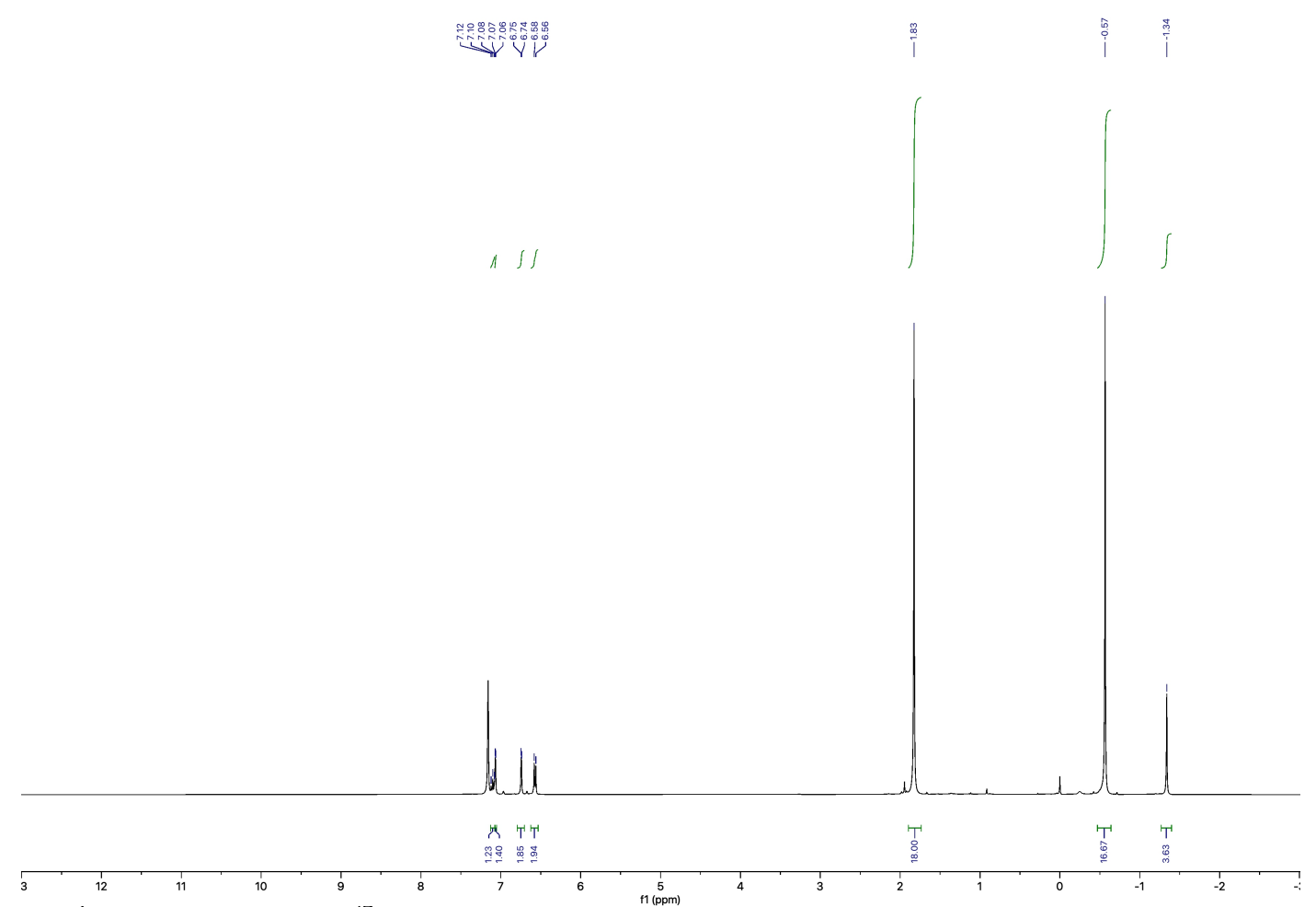

Figure S21. ${ }^{1} \mathrm{H}$ NMR spectrum of $\left({ }^{\mathrm{tBu}} \mathrm{ACNC}\right) \mathrm{Fe}\left(\mathrm{CH}_{2} \mathrm{SiMe}_{3}\right)_{2}(\mathrm{CO})$ in $\mathrm{C}_{6} \mathrm{D}_{6}$ at $25^{\circ} \mathrm{C}$.
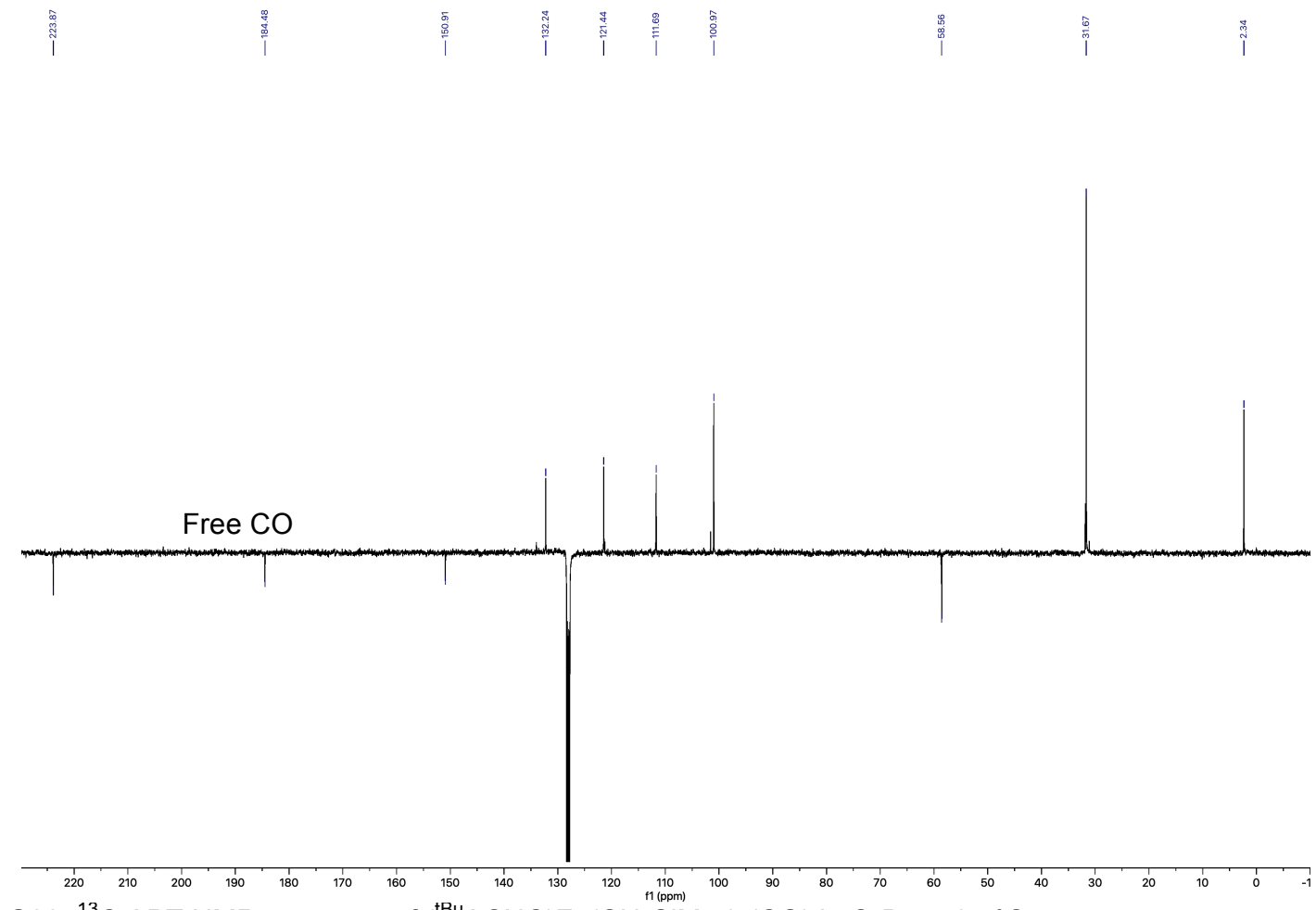

Figure S22. ${ }^{13} \mathrm{C}$ APT NMR spectrum of $\left({ }^{\text {tbu }} \mathrm{ACNC}\right) \mathrm{Fe}\left(\mathrm{CH}_{2} \mathrm{SiMe}_{3}\right)_{2}(\mathrm{CO})$ in $\mathrm{C}_{6} \mathrm{D}_{6}$ at $25^{\circ} \mathrm{C}$. 


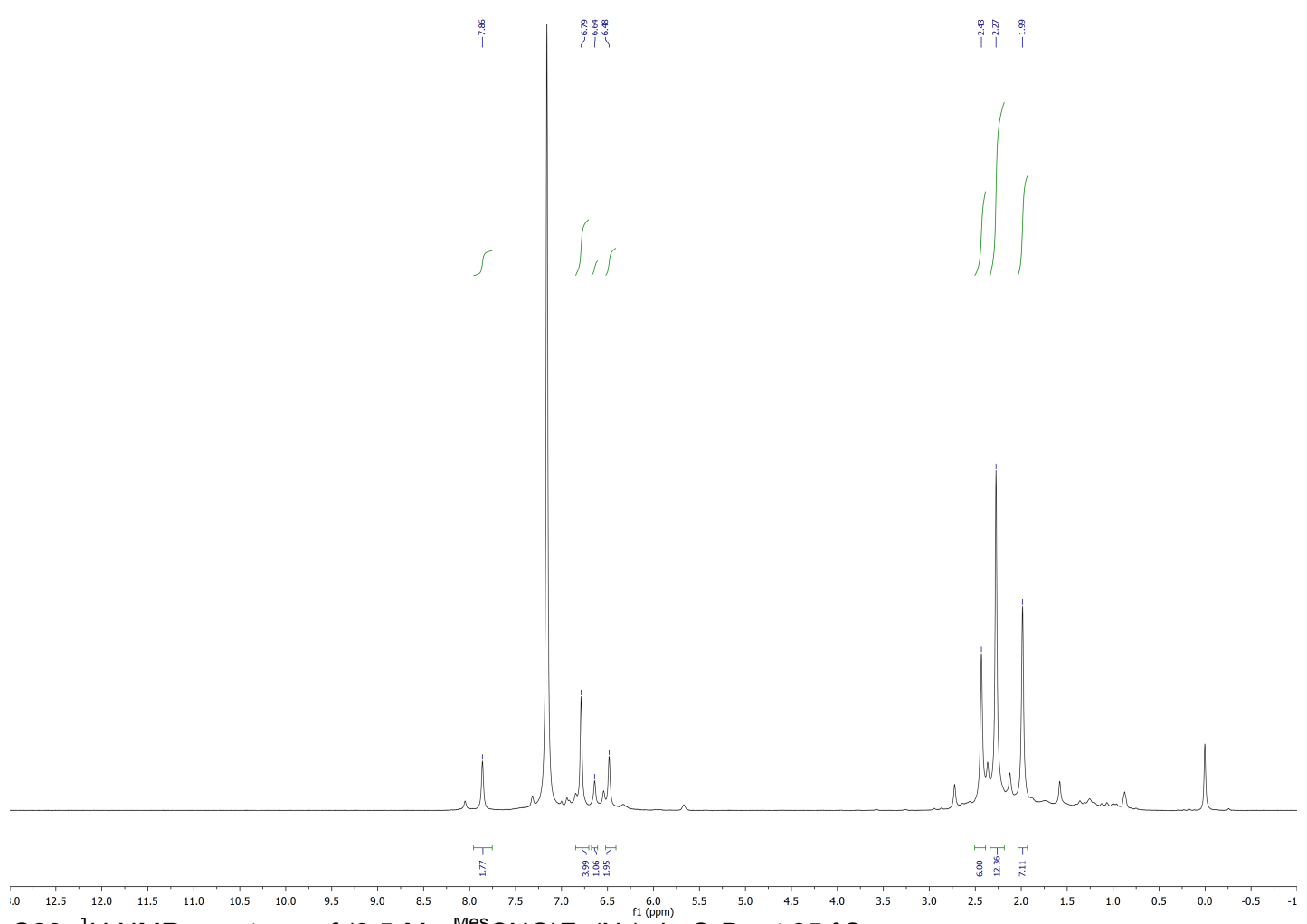

Figure S23. ${ }^{7} \mathrm{H}$ NMR spectrum of $\left(3,5-\mathrm{Me}_{2}{ }^{\mathrm{Mes}} \mathrm{CNC}\right) \mathrm{Fe}\left(\mathrm{N}_{2}\right)_{2}$ in $\mathrm{C}_{6} \mathrm{D}_{6}$ at $25^{\circ} \mathrm{C}$.

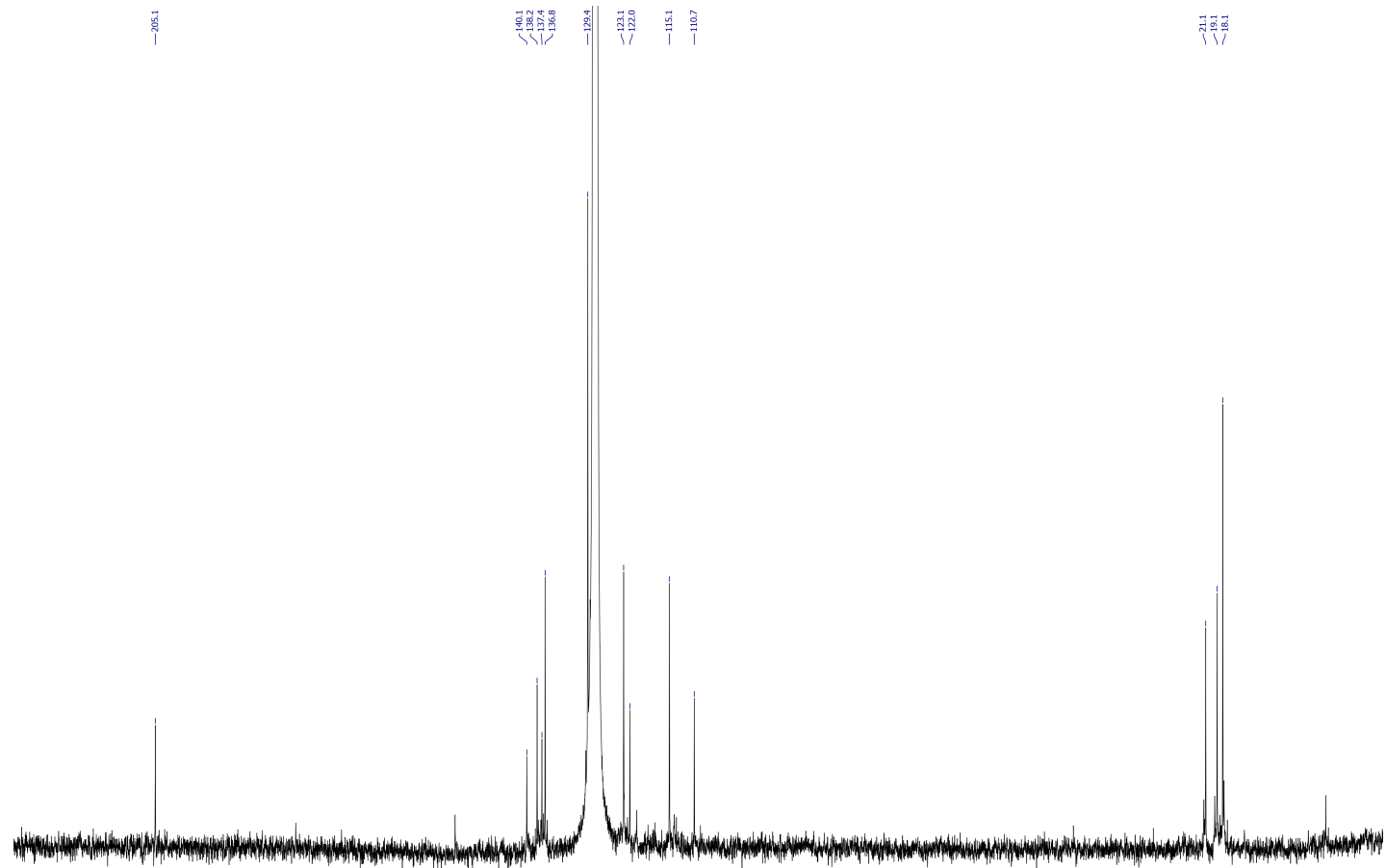

Figure S24. ${ }^{13} \mathrm{C}$ NMR spectrum of $\left(3,5-\mathrm{Me}_{2}{ }^{\mathrm{Mes}} \mathrm{CNC}\right) \mathrm{Fe}\left(\mathrm{N}_{2}\right)_{2}$ in $\mathrm{C}_{6} \mathrm{D}_{6}$ at $25^{\circ} \mathrm{C}$. 

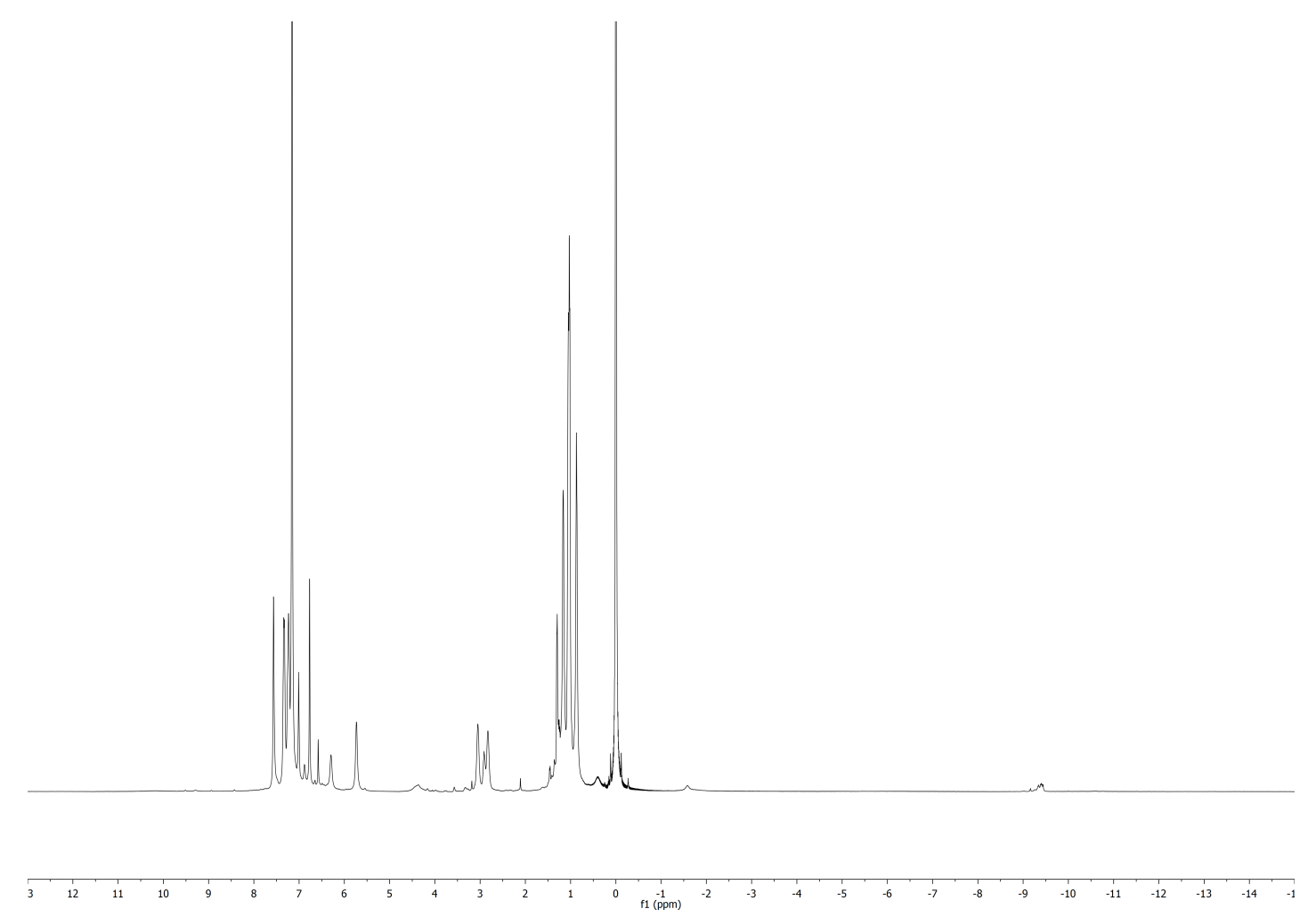

Figure S25. ${ }^{1} \mathrm{H}$ NMR spectrum of a reaction of $\left({ }^{\mathrm{PPr}} \mathrm{CNC}\right) \mathrm{Fe}\left(\mathrm{CH}_{2} \mathrm{SiMe}_{3}\right)_{2}\left(\mathrm{~N}_{2}\right)$ with $\mathrm{H}_{2}(4 \mathrm{~atm})$ in $\mathrm{C}_{6} \mathrm{D}_{6}$ after 10 min.

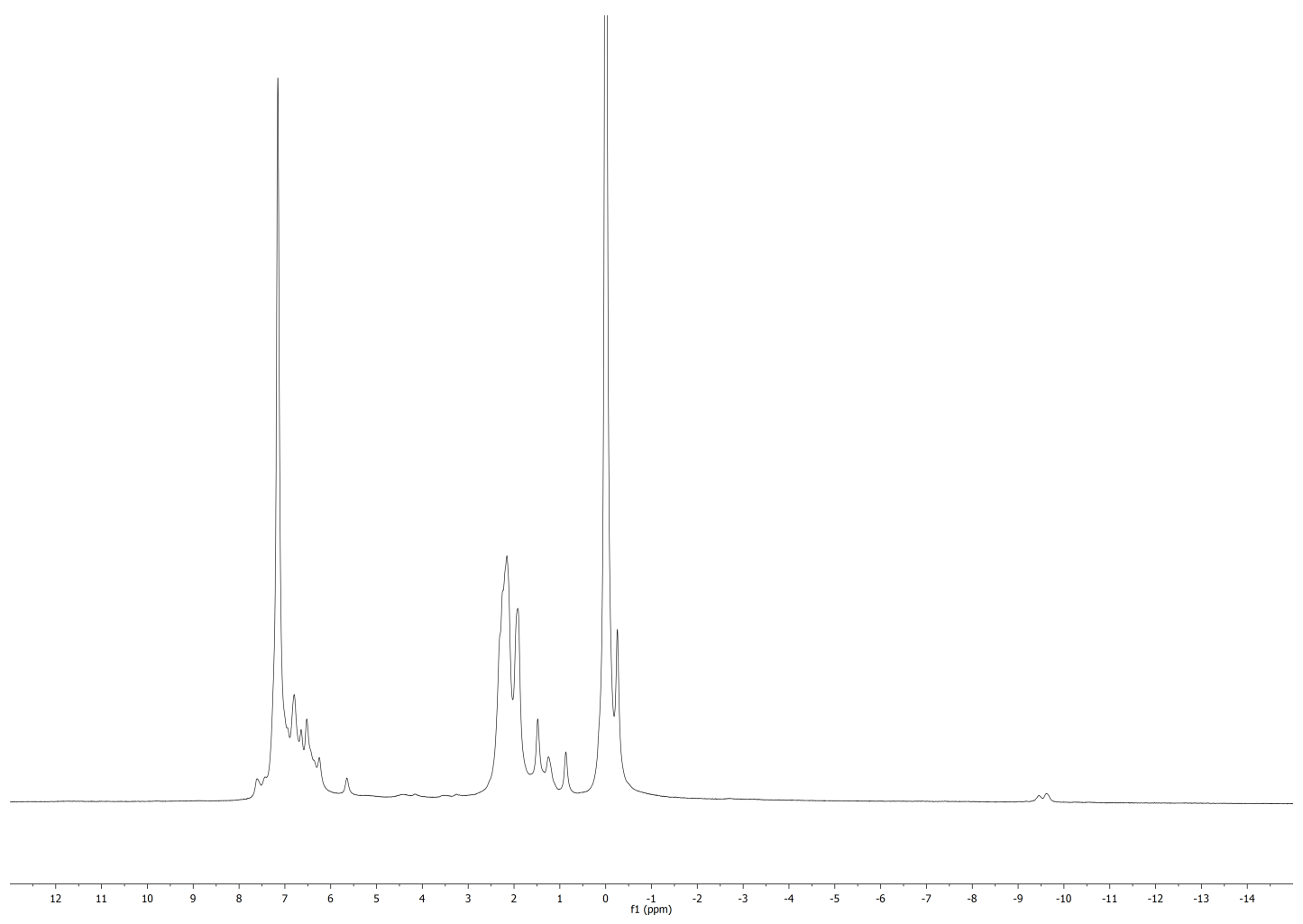

Figure S26. ${ }^{1} \mathrm{H}$ NMR spectrum of a reaction of $\left({ }^{\mathrm{Mes}} \mathrm{CNC}\right) \mathrm{Fe}\left(\mathrm{CH}_{2} \mathrm{SiMe}_{3}\right)_{2}\left(\mathrm{~N}_{2}\right)$ with $\mathrm{H}_{2}(4 \mathrm{~atm})$ in $\mathrm{C}_{6} \mathrm{D}_{6}$ after $10 \mathrm{~min}$. 


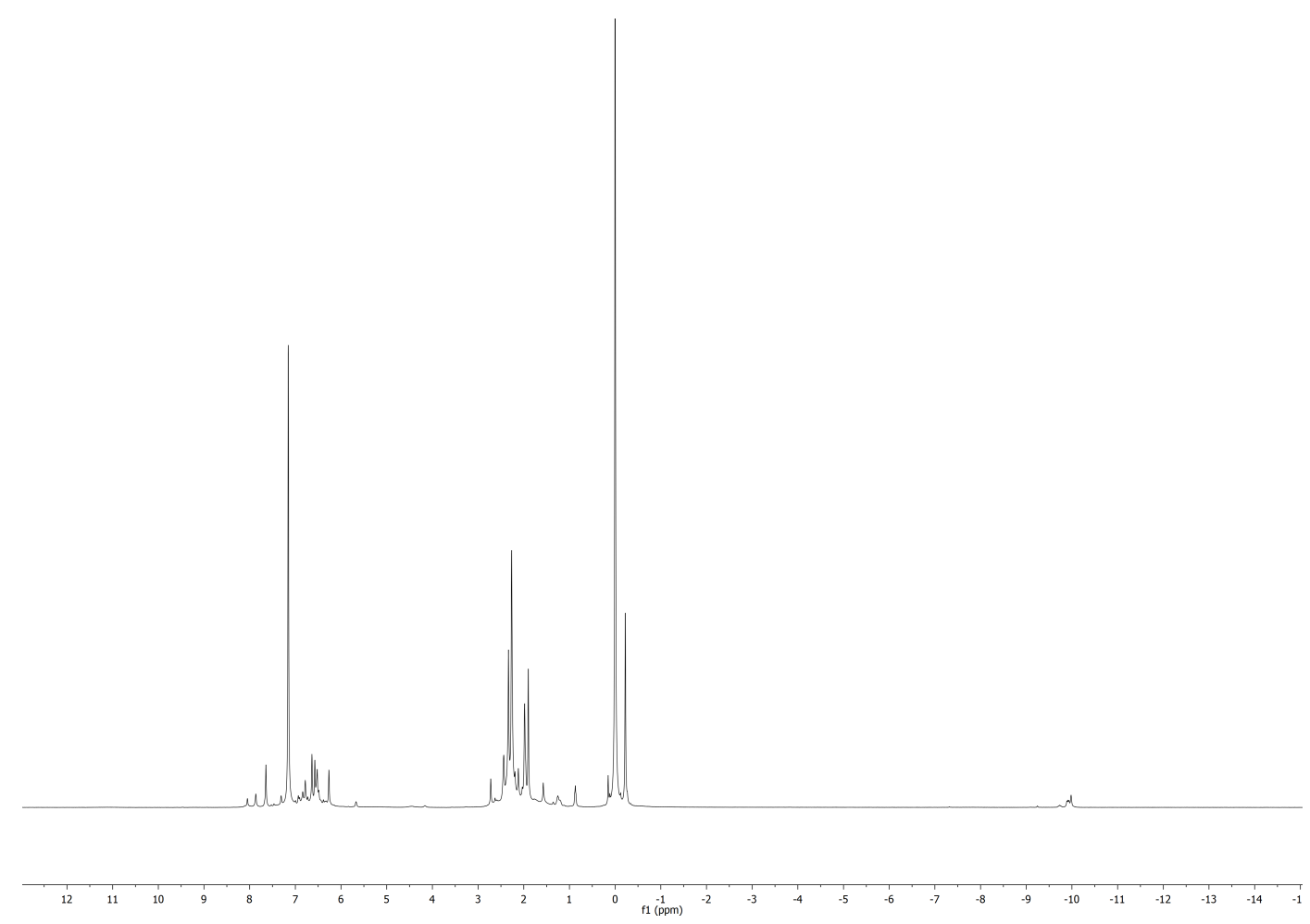

Figure S27. ${ }^{1} \mathrm{H}$ NMR spectrum of a reaction of $\left(3,5-\mathrm{Me}_{2}{ }^{\mathrm{Mes}} \mathrm{CNC}\right) \mathrm{Fe}\left(\mathrm{CH}_{2} \mathrm{SiMe}_{3}\right)_{2}\left(\mathrm{~N}_{2}\right)$ with $\mathrm{H}_{2}(4 \mathrm{~atm})$ in $\mathrm{C}_{6} \mathrm{D}_{6}$ after 10 min.

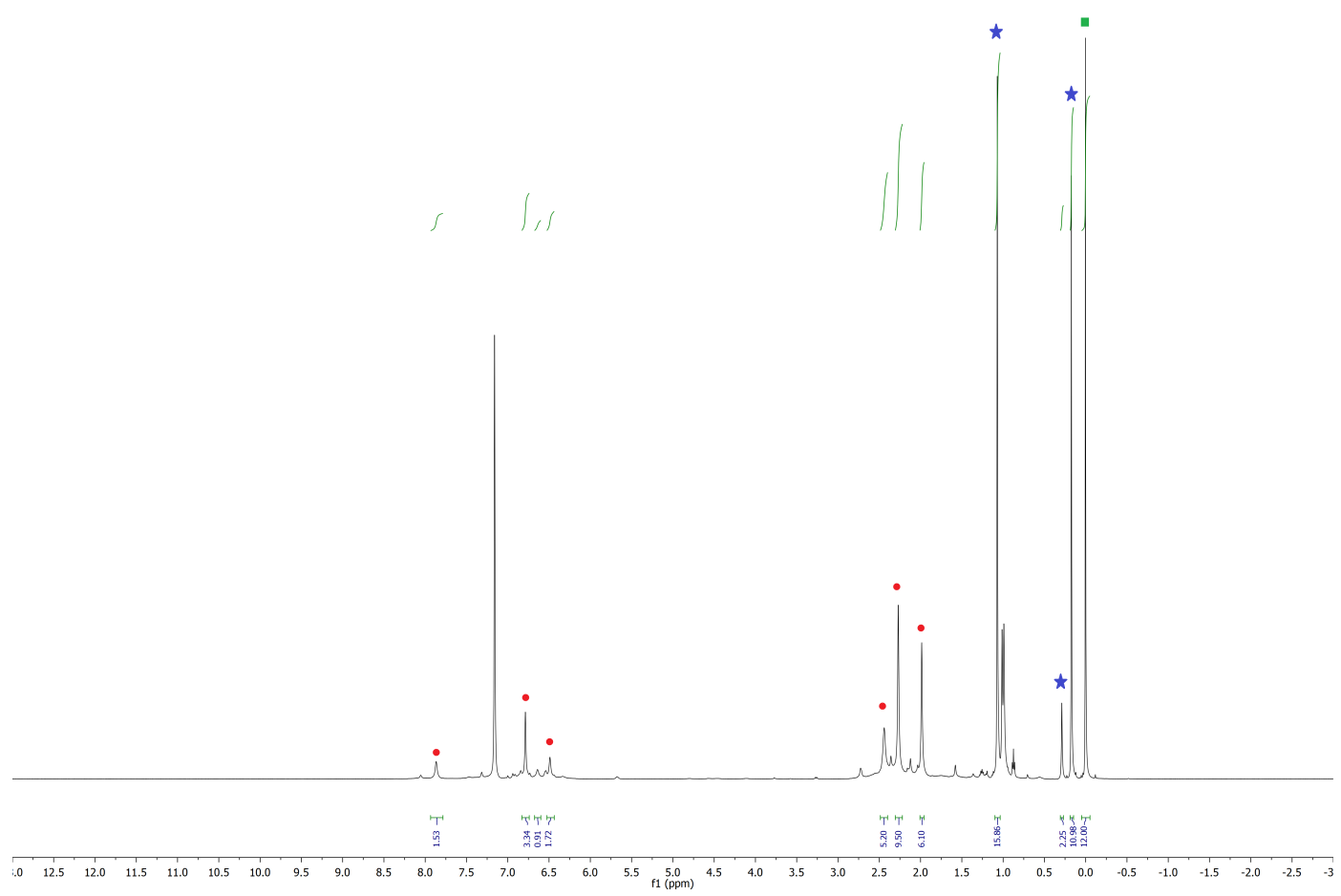

Figure S28. ${ }^{1} \mathrm{H}$ NMR spectrum of a mixture of $\left(3,5-\mathrm{Me}_{2}{ }^{\mathrm{Mes}} \mathrm{CNC}\right) \mathrm{Fe}\left(\mathrm{N}_{2}\right)_{2}$ (red circle), $\mathrm{SiMe}_{4}$ (green square), and $\mathrm{PinBCH}_{2} \mathrm{SiMe}_{3}$ (blue star) after a reaction of $\left(3,5-\mathrm{Me}_{2}{ }^{\mathrm{Mes}} \mathrm{CNC}\right) \mathrm{Fe}\left(\mathrm{CH}_{2} \mathrm{SiMe}_{3}\right)_{2}\left(\mathrm{~N}_{2}\right)$ with HBPin (1.5 equiv) in $\mathrm{C}_{6} \mathrm{D}_{6}$ after $10 \mathrm{~min}$. 


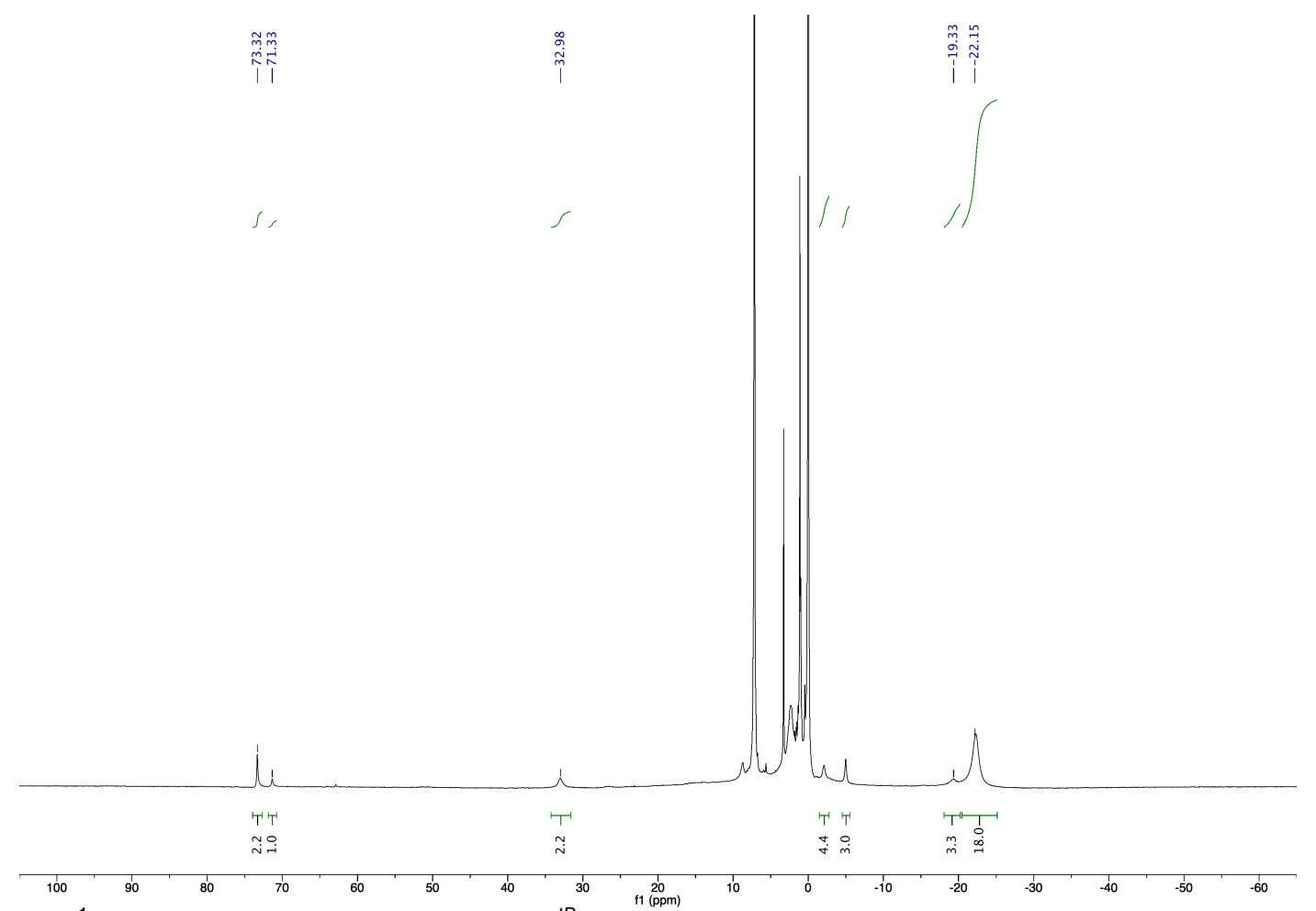

Figure S29. ${ }^{1} \mathrm{H}$ NMR spectrum of the reaction of $\left({ }^{\mathrm{tBu}} \mathrm{ACNC}\right) \mathrm{Fe}\left(\mathrm{CH}_{2} \mathrm{SiMe}_{3}\right)_{2}$ with $\mathrm{H}_{2}\left(4 \mathrm{~atm}\right.$.) after 10 minutes in $\mathrm{C}_{6} \mathrm{D}_{6}$ at $25^{\circ} \mathrm{C}$.

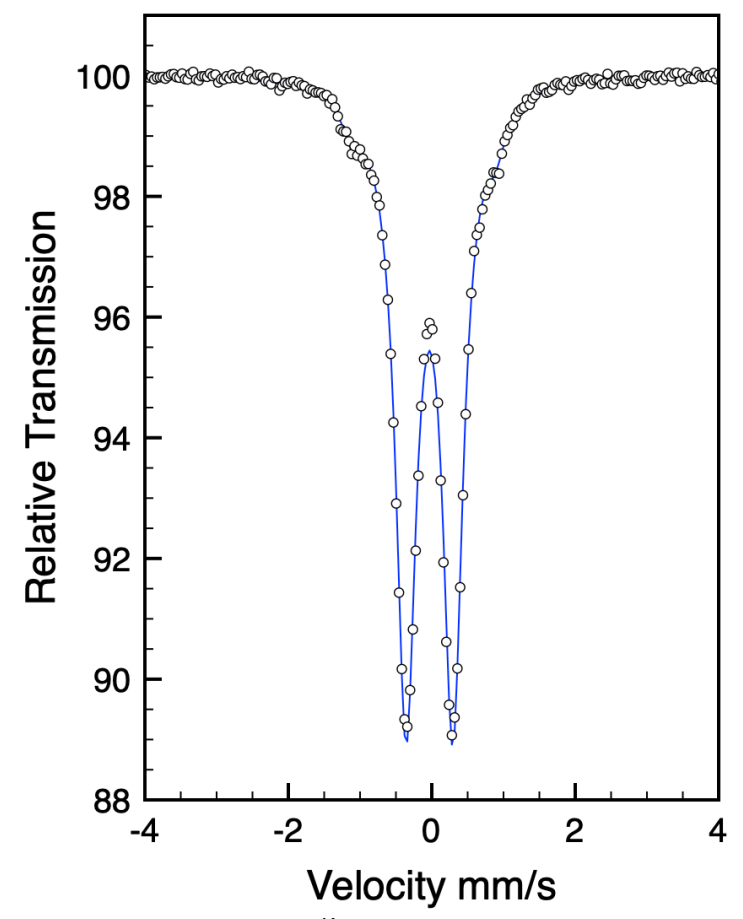

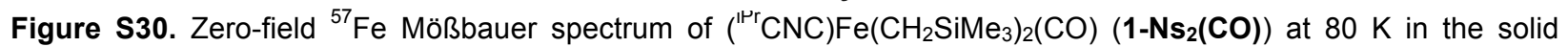
state. Simulated parameters (blue lines) are $\delta=-0.03 \mathrm{~mm} / \mathrm{s},\left|\Delta \mathrm{E}_{\mathrm{Q}}\right|=0.65 \mathrm{~mm} / \mathrm{s}$. 